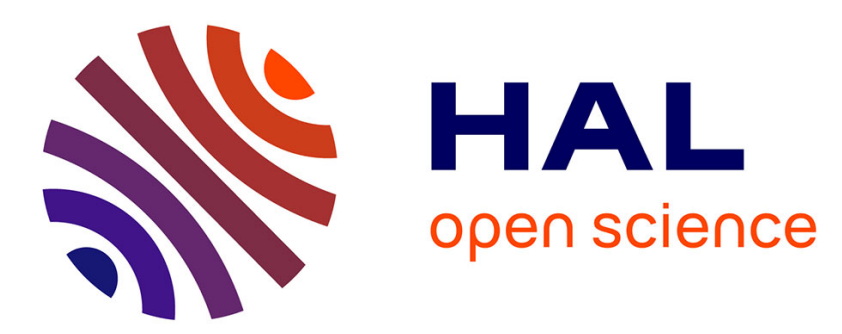

\title{
Bifurcations from steady to quasi-periodic flows in a laterally heated cavity filled with low Prandtl number fluids
}

\author{
A. Medelfef, D. Henry, A. Bouabdallah, S. Kaddeche
}

\section{- To cite this version:}

A. Medelfef, D. Henry, A. Bouabdallah, S. Kaddeche. Bifurcations from steady to quasi-periodic flows in a laterally heated cavity filled with low Prandtl number fluids. Journal of Fluid Mechanics, 2019, 861, pp.223-252. 10.1017/jfm.2018.912 . hal-02356287

\section{HAL Id: hal-02356287 \\ https://hal.science/hal-02356287}

Submitted on 13 Nov 2020

HAL is a multi-disciplinary open access archive for the deposit and dissemination of scientific research documents, whether they are published or not. The documents may come from teaching and research institutions in France or abroad, or from public or private research centers.
L'archive ouverte pluridisciplinaire $\mathbf{H A L}$, est destinée au dépôt et à la diffusion de documents scientifiques de niveau recherche, publiés ou non, émanant des établissements d'enseignement et de recherche français ou étrangers, des laboratoires publics ou privés. 


\title{
Bifurcations from steady to quasi-periodic flows in a laterally heated cavity filled with low-Prandtl number fluids
}

\author{
A. Medelfef ${ }^{1,2} \dagger$, D. Henry ${ }^{2}$, A. Bouabdallah ${ }^{1}$, and S. Kaddeche ${ }^{3}$ \\ ${ }^{1}$ Laboratoire de Thermodynamique et Systèmes Energétiques, Faculté de Physique, Université \\ des Sciences et de la Technologie Houari Boumediene - USTHB, BP 32, 16111 Bab Ezzouar, \\ Alger, Algérie \\ ${ }^{2}$ Laboratoire de Mécanique des Fluides et d'Acoustique, CNRS/Université de Lyon, Ecole \\ Centrale de Lyon/Université Lyon 1/INSA de Lyon - ECL, 36 avenue Guy de Collongue, \\ 69134 Ecully Cedex, France \\ ${ }^{3}$ Laboratoire de Recherche Matériaux, Mesures et Applications, Institut National des Sciences \\ Appliquées et de Technologie - INSAT, B.P. 676, 1080 Tunis Cedex, Tunisie
}

(Received $\mathrm{xx}$; revised $\mathrm{xx}$; accepted $\mathrm{xx}$ )

This study deals with the transition toward quasi-periodicity of buoyant convection generated by a horizontal temperature gradient in a three-dimensional parallelepipedic cavity with dimensions $4 \times 2 \times 1$ (length $\times$ width $\times$ height). Numerical continuation techniques, coupled with an Arnoldi method, are used to locate the steady and Hopf bifurcation points as well as the different steady and periodic flow branches emerging from them for Prandtl numbers ranging from 0 to 0.025 (liquid metals). Our results highlight the existence of two steady states along with many periodic cycles, all with different symmetries. The bifurcation scenarios consist of complex paths between these different solutions, giving a succession of stable flow states as the Grashof number is increased, from steady to periodic and quasi-periodic. The change of these scenarios with the Prandtl number, in connection with the crossing of bifurcation points, was carefully analysed.

Key words: Bifurcation, buoyant convection, low-Prandtl number.

\section{Introduction}

Buoyant convection driven by a horizontal temperature gradient is a canonical model of fluid mechanics, which can be used from a fundamental point of view to apprehend the development of instabilities and the transition to chaos and eventually turbulence. Besides this fundamental interest, the model is appropriate to study various natural, industrial and engineering problems, in domains such as geophysics (Hart 1972), renewable energies (Bacharoudis et al. 2007) or crystal growth (Lappa 2007). In crystal growth processes using the horizontal Bridgman technique, the melt, contained in a crucible withdrawn horizontally from a furnace, is subject to a horizontal temperature gradient generating convection. Very often, hydrodynamic instabilities in the melt will affect the quality of crystals, as they give rise to temperature fluctuations at the solidification front and lead to striations in the crystalline product (Dhanaraj et al. 2010; Pimputkar \& Ostrach 1981).

$\dagger$ Email address for correspondence: abs.medelfef@gmail.com 
Thus, there is considerable interest in understanding the development of the instabilities in such situations.

Very often, the studies are performed in finite size rectangular cavities, differentially heated between opposite vertical endwalls. In such cavities, the convective flow is governed by the temperature difference between the hot and cold endwalls (or, in a dimensionless form, by the Grashof number $G r$ ), the fluid ability to diffuse heat (the Prandtl number Pr, very small for molten metals) and the cavity dimensions (the aspect ratios).

Historically, the first studies have considered simplified situations, in which the aspect ratios in the horizontal directions tend to infinity. In this case, the Navier-Stokes equations admit a one-dimensional steady parallel flow solution, for which linear and non-linear stability analyses can be performed. Hart (1972) was the first to study this situation and he showed the existence of two types of instabilities (transverse steady shear instability or longitudinal oscillatory instability) depending on the Prandtl number. His stability study, which was linear and limited to small Prandtl numbers, was later extended by Laure (1987) and Laure \& Roux (1989) to include the non-linear analysis and by Gershuni et al. (1992) who considered higher Prandtl numbers and pointed out the existence of steady thermal instabilities in the case of perfectly conducting boundaries. These studies were a first step to understand the mechanisms of instability in differentially heated cavities. Nevertheless, as shown by Cormack et al. (1974a) through an asymptotic analysis of the two dimensional approximation, a more complete analysis is necessary because the real basic flow is not limited to the parallel flow part in the core region, but also includes non-parallel recirculating flows in the end regions.

Two dimensional simulations of the convective flows in such differentially heated cavities have then been carried out with the objective to understand the appearance and the development of time-dependent flows. Two different approximations have been used in this case:

- Some studies have simulated the flow in the vertical plane parallel to the gradient of temperature (in this case, the transverse dimension is supposed to be infinite or very large) (Cormack et al. 1974b; Pulicani et al. 1990; Mohamad \& Viskanta 1991; Henry \& Buffat 1998; Mercader et al. 2005). In these studies, different aspect ratios were considered and also different Prandtl numbers going from small Prandtl numbers to $\operatorname{Pr}=7$ (value for air). Note that in this approximation, the basic flow is no longer parallel and the recirculating flows at the endwalls will affect the results, particularly when the longitudinal aspect ratio is small.

- Other studies have supposed the longitudinal dimension to be infinite (or at least very large) and the flow to be invariant along this direction. In this approximation, the two-dimensional simulations concern the steady convective flows in the transverse crosssection plane (parallel to the isothermal cold and hot planes). Lyubimova et al. (2009a,b) performed the stability analysis of the basic flow obtained in this approximation. The critical instability thresholds are obtained for a large range of aspect ratios and Prandtl numbers, with and without magnetic field.

Concerning more realistic three dimensional cavities (length $L$, width $l$ and height $h$ ), several studies, on both experimental and numerical sides, were performed in order to understand the development of the convection, the appearance of instabilities and the transition scenarios leading to oscillatory flows.

The first leading experimental works are those of Hurle (Hurle 1966; Hurle et al. 1974). The experiments were performed in liquid gallium $(\operatorname{Pr} \approx 0.018)$ inside long open cavities having dimensions $10 \times 1.3 \times 1.5 \mathrm{~cm}^{3}, 10 \times 1 \times 1.5 \mathrm{~cm}^{3}$ and $10 \times 0.65 \times 1.5 \mathrm{~cm}^{3}$ $(L \times l \times h)$. The studies highlight the onset of oscillatory convection when the difference 
of temperature between the hot and the cold walls is increased. An extension of this work to study transition to more complicated time-dependent states was performed by McKell et al. (1990) in a $4 \times 1.3 \times 1 \mathrm{~cm}^{3}$ cavity in the presence of an applied magnetic field. The dynamics of the system is organized by a codimension- 2 bifurcation corresponding to the intersection of a line of secondary Hopf bifurcations with a line of period-doubling bifurcations. Other experimental studies concern large aspect ratio cavities. For instance, Hung \& Andereck (1988) used liquid mercury $(\operatorname{Pr} \approx 0.027)$ inside a cavity having dimensions $16.1 \times 16 \times 0.9 \mathrm{~cm}^{3}$. The results show that the first instability is connected with the oscillation of longitudinal rolls, which becomes noisy and eventually chaotic when the temperature difference is increased. While the measured wavelengths and frequencies are in agreement with the theoretical values of Hart (1972), the critical Grashof number is found to be higher than the expected theoretical thresholds. Daviaud \& Vince (1993) reported the observation of different dynamical regimes in silicone oil $(\operatorname{Pr} \approx 10)$ inside open cavities. For fixed horizontal dimensions $\left(20 \times 1 \mathrm{~cm}^{2}\right)$ and varying height $h$ of the fluid, Daviaud \& Vince (1993) observed that the first mode of instability is either longitudinal travelling waves (oscillatory modes) for $h<2.8 \mathrm{~mm}$ or steady transverse rolls for higher values of $h$ up to $10 \mathrm{~mm}$. Other experiments were also carried out by Mullin and co-workers (Braunsfurth \& Mullin 1996; Juel et al. 2001; Hof et al. 2004): the working fluid is the liquid gallium and the apparatus typically consists of an insulated $5 \times 1.3 \times 1 \mathrm{~cm}^{3}$ cavity differentially heated between the endwalls. Braunsfurth \& Mullin (1996) found that the transition to time-dependence takes place at supercritical Hopf bifurcations. Moreover, four different modes of oscillations are observed in a narrow $P r$ range $(0.016 \leqslant P r \leqslant 0.022)$. Juel et al. (2001) and Hof et al. (2004) then provided experimental and numerical results for three dimensional convection: the three dimensional properties of the flow as well as the transition towards oscillatory convection were pointed out on both experimental and numerical sides.

Three dimensional numerical simulations were also performed in order to understand the dynamics of the transition towards oscillatory convection. We can mention the studies of Dupont et al. (1987), Afrid \& Zebib (1990), Ben Hadid \& Henry (1997), Henry \& Buffat (1998), Wakitani (2000) and Henry \& Ben Hadid (2007), focused on low-Prandtl number situations. These numerical studies concern rather confined cavities with different typical aspect ratios and different boundary conditions (rigid or stress-free upper boundary). It was shown that the onset of oscillations is shifted toward higher values of the Grashof number when the upper surface is rather rigid than free and when the lateral confinement (in the transverse direction) is increased (stabilizing effect due to the viscous dissipation at the walls). For example, according to Afrid \& Zebib (1990), the threshold of oscillatory convection at $\operatorname{Pr}=0$ is equal to $G r=1.25 \times 10^{5}$ for a rigid top surface versus $1 \times 10^{5}$ for a stress-free upper boundary in a $4 \times 1 \times 1$ cavity $(L / h \times l / h \times h / h)$. And the threshold decreases to $G r=3 \times 10^{4}$ in a less confined rigid $4 \times 2 \times 1$ cavity. For a more extensive review on laterally heated situations in the context of crystal growth applications, we refer the reader to the work of Lappa (2007).

This work is an extension of the study of Henry \& Ben Hadid (2007), where the first instability thresholds in a differentially heated rigid cavity are determined for a wide range of longitudinal $(2<L / h<5)$ and transverse $(1<l / h<6)$ aspect ratios and for Prandtl numbers ranging from 0 to 0.03 . The first instability mode is found to be either steady or oscillatory depending on the Prandtl number and the aspect ratios. In particular, for a $4 \times 2 \times 1$ cavity, the first instability is triggered by a steady mode for $1 \times 10^{-4} \lesssim \operatorname{Pr} \lesssim 1.65 \times 10^{-2}$ and by an oscillatory mode for smaller or larger Prandtl numbers. In any case, energy budgets show that the destabilizing physical mechanism is the shear, principally due to the vertical variations of the longitudinal velocity. 


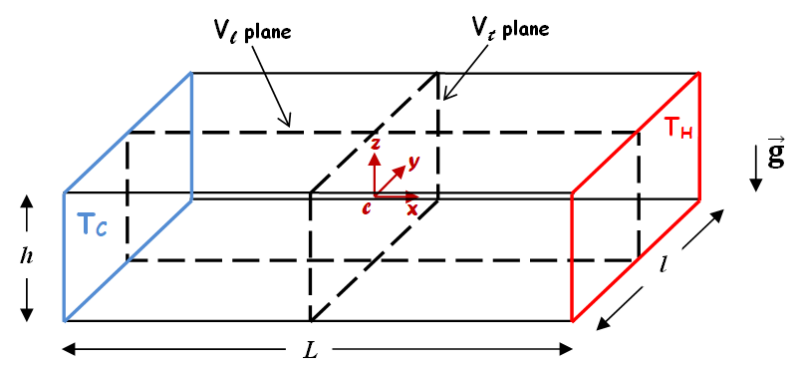

Figure 1: Geometry of the differentially heated cavity. The endwalls located at $\bar{x}=-L / 2$ and $\bar{x}=L / 2$ are held at fixed temperatures $T_{C}$ and $T_{H}$ (with $T_{H}>T_{C}$ ), respectively, whereas the sidewalls are insulating boundaries.

We focus here on the $4 \times 2 \times 1$ rigid cavity and on the low-Prandtl number range $(\operatorname{Pr} \in$ $[0,0.025])$. We want to understand how the oscillatory convection will develop, which type of oscillatory solution (periodic, quasi-periodic, with symmetry or not) can be observed, depending on the Prandtl number. These points need to be clarified, particularly in the intermediate range of Prandtl number where the first instability is steady. To tackle this problem, a spectral element code is used allowing time evolution calculations, the continuation of steady solutions, but also the newly developed continuation of periodic oscillatory solutions. Note that our idea is not to simulate a real experiment, for which the boundary conditions are difficult to assess and which would require variable fluid properties with the temperature, but to give valuable information on the dynamics of the flow in such a situation.

In the following, the mathematical model governing our physical problem as well as the numerical methods used to solve it are first presented. The steady basic flow at low Gr (before the onset of instabilities), its structure, its symmetry properties are then described. The main results of our simulations are finally presented: the steady solutions and the different bifurcation points along them, the oscillatory periodic or quasi-periodic solutions. The solutions obtained are first described as a function of the Grashof number for characteristic Prandtl numbers, before giving a more general view in the whole Prandtl number range.

\section{Physical model and numerical methods}

In this paper, we consider the flow in a differentially heated parallelepipedic cavity of aspect ratios $A_{X}=L / h=4$ and $A_{Y}=l / h=2$, where $L$ is the length along the longitudinal $x$-direction, $l$ is the width along the transverse $y$-direction and $h$ is the height along the vertical $z$-direction (see Fig. 1, where the main middle vertical planes, either longitudinal ( $V_{l}$ plane) or transverse $\left(V_{t}\right.$ plane) are presented). The vertical endwalls are isothermal and held at different temperatures, $T_{H}$ at the right hot endwall and $T_{C}$ at the left cold endwall, and the sidewalls are insulating. The fluid is assumed to be Newtonian with constant physical properties (kinematic viscosity $\nu$, thermal diffusivity $\kappa$, density $\rho$ ), except for the density in the buoyancy term, which, in the Boussinesq approximation, depends linearly on the temperature, $\rho=\rho_{0}\left(1-\beta\left(T-T_{0}\right)\right)$, where $\beta$ is the thermal expansion coefficient, $T_{0}=\left(T_{C}+T_{H}\right) / 2$ is a reference temperature, and $\rho_{0}$ is the value of the density at $T_{0}$.

The convective motion in the differentially heated cavity is governed by the momentum, mass and energy conservation equations. By using $h, h^{2} / \nu, \nu / h, \rho_{0} \nu^{2} / h^{2}$ and $\Delta T / A_{X}$ 
$\left(\Delta T=T_{H}-T_{C}\right)$ as reference quantities for length, time, velocity, pressure and temperature, respectively, the equations take the following dimensionless form:

$$
\begin{gathered}
\frac{\partial \boldsymbol{v}}{\partial t}+\boldsymbol{v} \cdot \boldsymbol{\nabla v}=-\nabla p+\nabla^{2} \boldsymbol{v}+G r \Theta \boldsymbol{e}_{z} \\
\frac{\partial \Theta}{\partial t}+\boldsymbol{v} \cdot \nabla \Theta=\frac{1}{P r} \nabla^{2} \Theta \\
\boldsymbol{\nabla} \cdot \boldsymbol{v}=0
\end{gathered}
$$

where $G r=g \beta h^{3} \Delta T /\left(A_{X} \nu^{2}\right)$ is the Grashof number, $\operatorname{Pr}=\nu / \kappa$ is the Prandtl number and $\Theta=\left(T-T_{0}\right) / \Delta T$ is a reduced temperature. Note that the origin of the coordinates is taken at the center $C$ of the cavity.

The boundary conditions are $\boldsymbol{v}=0$ (no-slip conditions) for the velocity on all the boundaries, $(\partial \Theta / \partial z)(z= \pm 1 / 2)=0$ and $(\partial \Theta / \partial y)\left(y= \pm A_{Y} / 2\right)=0$ for the temperature on the insulating sidewalls, and $\Theta\left(x= \pm \frac{A_{X}}{2}\right)= \pm \frac{A_{X}}{2}$ for the temperature on the isothermal endwalls.

Equations (2.1), (2.2) and (2.3) are solved in the three-dimensional domain using a spectral element code developed by Ben Hadid \& Henry (1997). This code is based on a spatial discretization using the Gauss-Lobatto-Legendre polynomials. The time discretization is carried out by a semi-implicit scheme proposed by Karniadakis (1991), where the non-linear terms are included explicitly, the pressure is then obtained from a derived pressure equation enforcing the incompressibility constraint (with consistent boundary conditions) and finally the linear terms are integrated implicitly.

The time integration scheme is used with the third order accurate formulation proposed by Karniadakis (1991) for transient computations or unsteady flow simulations. It is also used in its first order formulation to compute steady state solutions, eigenvalues and eigenvectors determining their stability, and bifurcation points. All these computations are based on a Newton-Krylov method and follow the ideas of Mamun \& Tuckerman (1995) and Bergeon et al. (1998). They are integrated in a continuation algorithm and, complemented by an Arnoldi method, they allow to obtain bifurcation diagrams of the steady flow solutions. These methods will not be described further here as they have been already presented and well discussed by Henry \& Ben Hadid (2007) and Torres et al. $(2013,2014)$. In these papers, the methods were successfully used in different problems of buoyant convection.

The continuation of periodic orbits (or cycles) has also been developed more recently using the method proposed by Sánchez et al. (2004) and successfully used by Puigjaner et al. (2011) in a Rayleigh-Bénard problem. The novelty here is that the method is developed from a time integration scheme using time splitting. The method is based on a Newton-Krylov approach in which the periodic states of Eqs. (2.1-2.3) are obtained as fixed points of a Poincaré map. In our code, the hyperplane defining the Poincaré map corresponds to a given value $k_{p}$ for $u^{*}$, the longitudinal velocity at a chosen point inside the cavity, i.e. $u^{*}=k_{p}$. The trajectories in the phase space used to approach the periodic state are computed with the time integration scheme at third order with a time step $\Delta t_{c}=510^{-5}$. These trajectories are initiated from a point in the Poincaré hyperplane using two first-order time steps with $\Delta t=\Delta t_{c} / 4$ and three second-order time steps with $\Delta t=\Delta t_{c} / 2$, until third-order time steps with $\Delta t=\Delta t_{c}$ can be applied. The exact return of the trajectory to the Poincaré hyperplane is obtained by a Newton-Krylov approach as proposed by Sánchez et al. (2004). Finally, the successive corrections of the initial point in the Poincaré hyperplane, necessary to converge to a closed loop (the expected cycle), are obtained, as indicated above, by Newton-Krylov steps. As shown by Sánchez 
et al. (2004), at each Newton-Krylov step, the linear system giving the correction can be solved by GMRES iterations, where the matrix-vector products correspond to the evolution of a perturbation obtained by iterating the linearized version of Eqs. (2.1-2.3) along the last calculated trajectory in the phase space. The correction thus obtained at a Newton-Krylov step is eventually adapted to lie in the Poincaré hyperplane. The convergence to the periodic solution is assumed to be obtained when the mean square difference between the initial and end points in the Poincaré hyperplane is less than $10^{-6}$. The method was found to work well with a convergence generally obtained with a few Newton-Krylov steps (2 to 4), each Newton-Krylov step requiring 5 to 15 GMRES iterations for a prescribed precision of $10^{-2}$.

The stability of these periodic solutions is investigated in the framework of the Floquet theory (Klausmeier 2008; Seydel 2010). We use an Arnoldi method in which the Arnoldi basis is obtained by repeating the same process as for the GMRES iterations, i.e. computing the evolution of a perturbation by iterating the linearized version of Eqs. (2.1-2.3) along the considered periodic solution. The complex eigenvalues $\sigma$ obtained by the Arnoldi method are the Floquet multipliers. If the norm of $\sigma$ is smaller (larger) than 1, i.e. the Floquet multiplier is inside (outside) the unit circle, the perturbation associated with the corresponding eigenvector will decrease (increase) during the cycle, indicating a stabilizing (destabilizing) behaviour. Note, however, that there is always a Floquet multiplier equal to $(+1)$, corresponding to a perturbation exactly along the cycle, which will neither decrease nor increase. Excluding this last eigenvalue, the periodic solution will be unstable if there is at least one Floquet multiplier outside the unit circle (i.e. $\exists j,\left|\sigma_{j}\right|>1$ ). Conversely, if all the Floquet multipliers are inside the unit circle (i.e. $\forall j,\left|\sigma_{j}\right|<1$ ), the periodic solution is stable. Finally, depending on how these Floquet multipliers cross the unit circle, three kinds of bifurcation can be obtained. If the unstable Floquet multiplier is real, a period doubling bifurcation will occur for $\sigma=-1$ and a bifurcation toward another limit cycle with the same frequency for $\sigma=+1$. In contrast, if the unstable Floquet multiplier is a pair of complex conjugate eigenvalues, a Naimark-Sacker bifurcation (also called generalized Hopf bifurcation) toward a quasiperiodic solution is identified. In practice, the ten leading Floquet multipliers were calculated with the Arnoldi method and the precise convergence $\left(10^{-10}\right)$ was obtained within a limited number of Arnoldi steps (50 to 60).

The refined grid used for all our calculations of convective flow in a cavity with aspect ratios $A_{X}=4$ and $A_{Y}=2$ has $47 \times 37 \times 27$ points in the $x, y$ and $z$ directions, respectively. As shown in Table 1 on some examples, this grid gives excellent resolution of the thresholds $G r_{c}$ of the different bifurcation points, indicating that both the solution and the eigenvector that will induce the oscillatory behaviour are well resolved on the grid.

\section{Steady flow at low Grashof numbers}

In our differentially heated parallelepipedic cavity, a convective flow will appear as soon as the Grashof number $G r$ is not zero. At low $G r$, this flow is steady, unicellular and occupies the whole enclosure. More precisely, the fluid rises at the hot wall, travels longitudinally throughout the cavity and goes down at the cold wall, before returning to the hot wall (Fig. 2). As long as the temperature field remains diffusive and the inertial effects remain small (roughly up to $G r=1000$ ), the flow structure is invariant, with in particular a quasi-parallel flow in the core of the cavity (Hadley flow, see Hart (1972)), and only its intensity varies linearly with $G r$. In the Boussinesq approximation, this 


$\begin{array}{cccc}N_{X} \times N_{Y} \times N_{Z} & G r_{c}\left(P_{l}^{P}\right) & G r_{c}\left(H_{a}^{P}\right) & \omega_{c}\left(H_{a}^{P}\right) \\ 41 \times 31 \times 21 & 28955.25 & 30573.90 & 221.09 \\ 43 \times 33 \times 23 & 28954.80 & 30571.30 & 221.08 \\ 45 \times 35 \times 25 & 28959.58 & 30569.23 & 221.07 \\ 47 \times 37 \times 27 & 28957.50 & 30569.53 & 221.08 \\ 49 \times 39 \times 29 & 28957.40 & 30569.68 & 221.08 \\ 51 \times 41 \times 31 & 28957.66 & 30569.52 & 221.08 \\ 53 \times 43 \times 33 & 28957.54 & 30569.40 & 221.08\end{array}$

Table 1: Mesh refinement tests of numerical accuracy of the critical Grashof number $G r_{c}$ for different flow bifurcations detected in a laterally heated cavity $\left(A_{X}=4, A_{Y}=2\right.$, $\operatorname{Pr}=0.005)$ : steady bifurcation $P_{l}^{P}$ and Hopf bifurcation $H_{a}^{P}$ for which the value of $\omega_{c}$ is also given.

steady flow exhibits different symmetries coming from the symmetries of the equations, the geometry and the boundary conditions:

- a reflection symmetry $S_{l}$ with respect to the middle vertical $V_{l}$ plane $(y=0)$, also called left/right symmetry:

$$
S_{l}:(x, y, z, t) \rightarrow(x,-y, z, t), \quad(u, v, w, \Theta) \rightarrow(u,-v, w, \Theta),
$$

- a $\pi$-rotational symmetry $S_{\pi}$ with respect to the central transverse $y$-axis $(x=0$, $z=0)$ :

$$
S_{\pi}:(x, y, z, t) \rightarrow(-x, y,-z, t), \quad(u, v, w, \Theta) \rightarrow(-u, v,-w,-\Theta),
$$

- a symmetry $S_{c}$ with respect to the center $C$ of the cavity, which is such that:

$$
S_{c}=S_{\pi} \cdot S_{l}
$$

These symmetries belong to a $Z_{2} \times Z_{2}=D_{2}$ group, which contains four elements (including the identity $I$ ). Note that we will also use the notations $S_{a}$ and $S_{w}$ for flows with all the symmetries and without symmetry, respectively.

The plot of the longitudinal velocity component in the transverse $V_{t}$ plane, $u(y, z)$, given in Fig. 2a allows to clearly visualize these symmetries. Indeed, for $u$ in this plane, the $S_{\pi}$ symmetry is expressed by $(y, z) \rightarrow(y,-z), u \rightarrow-u$, the $S_{l}$ symmetry is given by $(y, z) \rightarrow(-y, z), u \rightarrow u$ and finally, the $S_{c}$ symmetry is such that $(y, z) \rightarrow(-y,-z)$, $u \rightarrow-u$. Such a plot can then be used to see the symmetries of solutions on new branches appearing at bifurcation points. Applied on a critical eigenvector $u^{\prime}(y, z)$ (Fig. 5), the plot will also indicate which symmetries are kept or broken (in that case, they appear as anti-symmetries) at the corresponding bifurcation point.

The changes which affect the steady three dimensional flow structure (shown in Fig. 2 before any symmetry breaking at bifurcations) when the Grashof number is increased are described in Henry \& Buffat (1998) and Henry \& Ben Hadid (2007). At Gr =10000, the uni-cellular flow appears to be already tilted compared to the parallel Hadley circulation. For larger values of $G r$, the uni-cellular flow evolves towards a centered roll-like structure inside a remaining long-scale circulation. As indicated by Henry \& Buffat (1998), these changes are the sign of an imperfect bifurcation which is connected with the steady transition towards transverse rolls (shear instability) found in the stability analysis of the parallel Hadley flow (Hart 1972). At sufficiently high values of the Grashof number, 
(a)

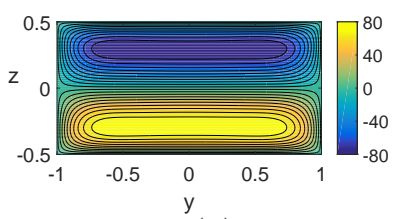

(b)

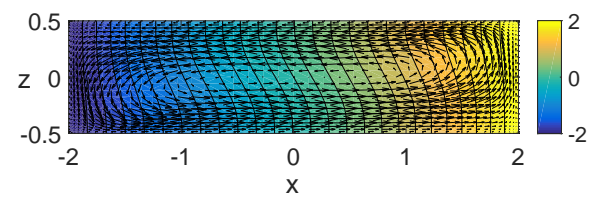

(c)

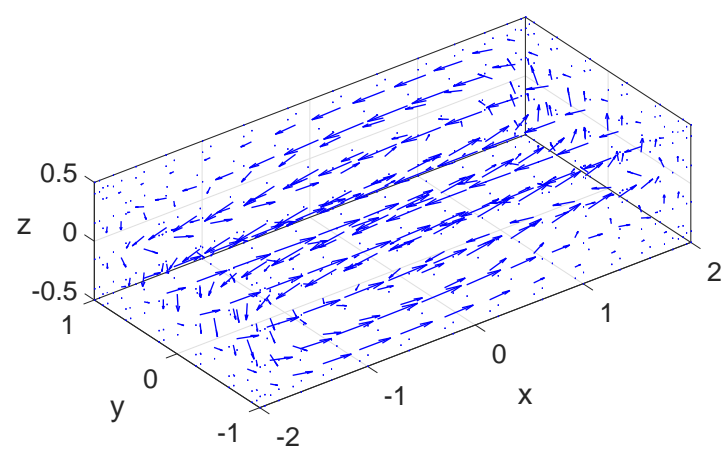

Figure 2: For $\operatorname{Pr}=0.025$ and $G r=3 \times 10^{4}$ : (a) Longitudinal velocity field $u(y, z)$ in the $V_{t}$ plane; (b) Velocity vector $(u, w)$ and temperature $-2 \leqslant \Theta(x, z) \leqslant 2$ fields in the $V_{l}$ plane; (c) A three dimensional visualisation of the velocity vector field showing the circulation loop in the cavity.

this basic steady flow will eventually be destabilized at different bifurcation points, which will trigger new steady or oscillatory flow solutions. This is discussed in the next sections.

\section{Bifurcation analysis of the steady flows}

In a previous work, Henry \& Ben Hadid (2007) determined the threshold of the first instability of the basic steady flow in a differentially heated parallelepipedic cavity for a wide range of aspect ratios $A_{X}$ and $A_{Y}$ and of Prandtl numbers. They showed the strong variation of these thresholds, which were associated with instabilities of different types (steady or oscillatory), breaking different symmetries. In the present study, the cavity dimensions are held constant $\left(A_{X}=4\right.$ and $\left.A_{Y}=2\right)$ and only the effect of the Prandtl number will then be depicted. However, we will not focus on the first instability of the basic steady flow, but more largely consider all the bifurcation points that could be involved in the further dynamics of the flow, on the basic steady flow branch (primary branch) as well as on the steady branches that bifurcate from it (secondary branches). Note that, in the different bifurcation diagrams presented in this study, we plot $u^{*}$, 


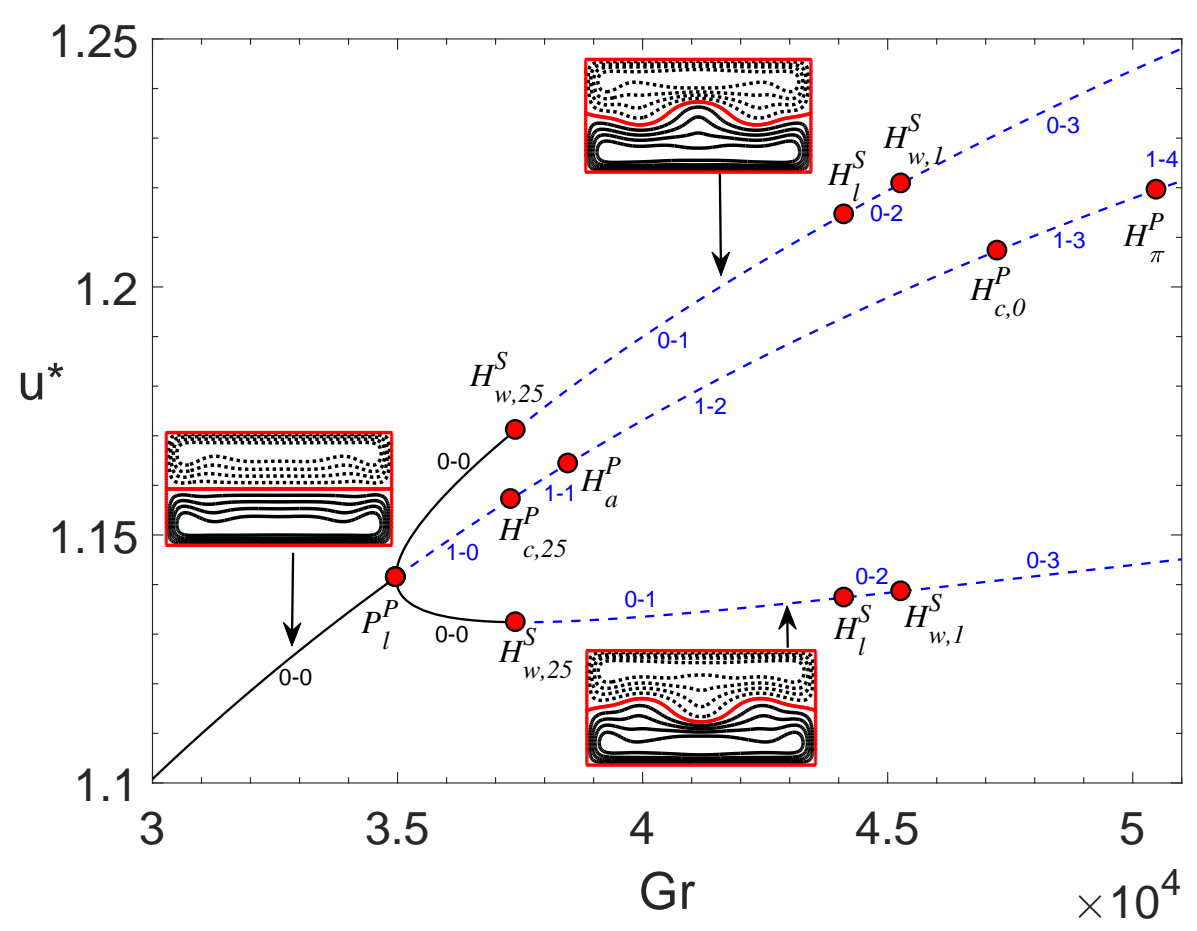

Figure 3: Bifurcation diagram $\left(u^{*}\right.$ versus $\left.G r\right)$ for $\operatorname{Pr}=0.015$ showing all the bifurcation points in the range $3.0 \times 10^{4} \leqslant G r \leqslant 5.1 \times 10^{4}$. Stable steady solutions are represented by solid lines whereas dashed lines represent unstable steady solutions. The symmetry of these steady solutions ( $S_{a}$ for the leading branch and $S_{l}$ for the secondary branch) is shown in the insets. More information on the stability is given by the number of unstable eigenvalues $n-m$ indicated along the branches (see text).

the normalized longitudinal velocity component $(u / \sqrt{G r})$ at a representative point in the cavity $(x=0, y=0.49391, z=-0.36911)$, as a function of $G r$. Finally, the stability of the branches (branches of steady states as well as branches of cycles) on these bifurcation diagrams is indicated through a couple of numbers $n-m$ where $n$ is the number of unstable real eigenvalues whereas $m$ is the number of unstable couples of complex conjugate eigenvalues. $0-0$ is thus the indication of a stable branch.

As a starting point, we have computed bifurcation diagrams of the steady flow solutions for several Prandtl numbers going from 0 to 0.025 (see Fig. 3 and the steady part of the different bifurcation diagrams in Figs. 6a, 7a, 8a, 10a). In all these cases, a single steady bifurcation point $P_{l}^{P}$ is found on the primary branch (see Table 2). As shown in Fig. 3 , this bifurcation is a supercritical pitchfork bifurcation which breaks the $S_{c}$ and $S_{\pi}$ symmetries and keeps the $S_{l}$ symmetry. The secondary branch initiated at this point thus corresponds to flow states with only the $S_{l}$ symmetry (see insets of Fig. 3 for example). Note that, as expected, two secondary branches appear at the pitchfork bifurcation. These branches are dynamically equivalent as the solution on one branch is the symmetric of the solution on the other branch by the broken $S_{c}$ or $S_{\pi}$ symmetry. For this reason, only one of these branches will often be considered and plotted. The principal bifurcation points on these steady primary and secondary branches are also determined. Except $P_{l}^{P}$, they are all Hopf bifurcation points (see Fig. 3) and their critical characteristics (critical 


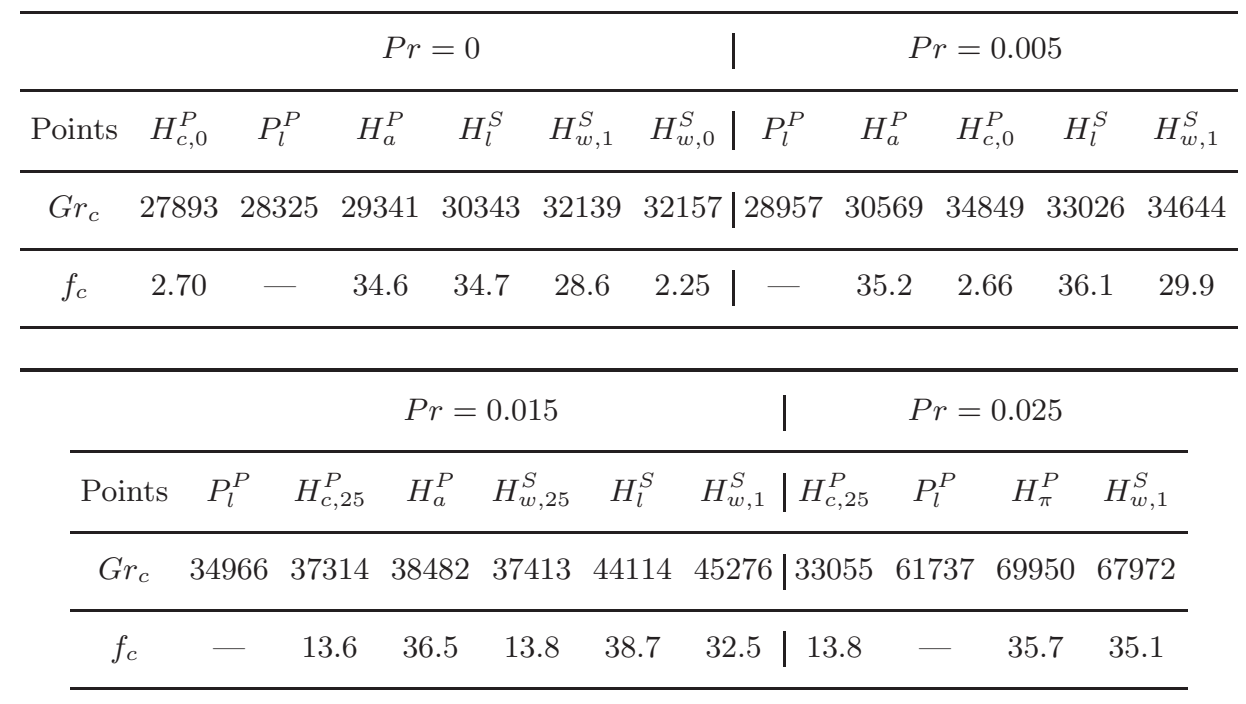

Table 2: Critical thresholds $G r_{c}$ of the principal bifurcation points ( $P$ for pitchfork, $H$ for Hopf) on the primary (superscript $P$ ) and secondary (superscript $S$ ) branches for $P r=0$, $0.005,0.015$ and 0.025 . The subscripts $c, l, \pi, a$ and $w$ refer to the symmetries kept by the marginal mode at the bifurcation: symmetries $S_{c}, S_{l}, S_{\pi}$, all the symmetries and without symmetry, respectively. In case of Hopf bifurcation points, the critical frequency at threshold $f_{c}$ is also given.

threshold $G r_{c}$ and frequency $f_{c}$ ) are given in Table 2. In order to identify all the different bifurcation points, we have given them specific names depending on their properties. These points are either steady pitchfork $(P)$ or oscillatory ( $H$ for Hopf). The type of the branch (primary or secondary) on which they appear is indicated by a superscript ( $P$ for primary branch and $S$ for secondary branch), whereas the symmetry preserved by the critical mode at the bifurcation is mentioned by a subscript. More precisely, the subscripts $c, l$ and $\pi$ correspond to the centro-symmetry $S_{c}$, the left/right symmetry $S_{l}$ and the $\pi$-rotational symmetry $S_{\pi}$, respectively, whereas the subscript $a(w)$ indicates critical modes with all the symmetries (without symmetry). For example, the points $H_{a}^{P}$ and $H_{l}^{S}$ are the Hopf bifurcation point on the primary branch for which all the symmetries are kept and the Hopf bifurcation point on the secondary branch that keeps only the $S_{l}$ symmetry, respectively. The same type of notations will be used later for the bifurcations occurring on cyles: the superscript $P$ or $S$ will be changed to $C$ to mention that the bifurcation occurs on a cycle, but with a further indication on the symmetry of the cycle. For example the superscript $C_{a}$ will indicate that the bifurcation occurs on a cycle with all the symmetries. The subscript will still indicate the symmetry preserved by the critical mode.

From Fig. 3 and Tab. 2, we can see that five main modes were found on the primary steady branch $\left(P_{l}^{P}, H_{c, 0}^{P}, H_{c, 25}^{P}, H_{a}^{P}\right.$ and $\left.H_{\pi}^{P}\right)$ and four other modes $\left(H_{l}^{S}, H_{w, 0}^{S}, H_{w, 1}^{S}\right.$ and $H_{w, 25}^{S}$ ) on the secondary steady branch initiated at the steady point $P_{l}^{P}$. All these bifurcation points were then precisely tracked as a function of $\mathrm{Pr}$ in the whole range $0 \leqslant \operatorname{Pr} \leqslant 0.025$. The location of these points in the $\left(\operatorname{Pr}, G r_{c}\right)$ parameter space is shown 
(a)

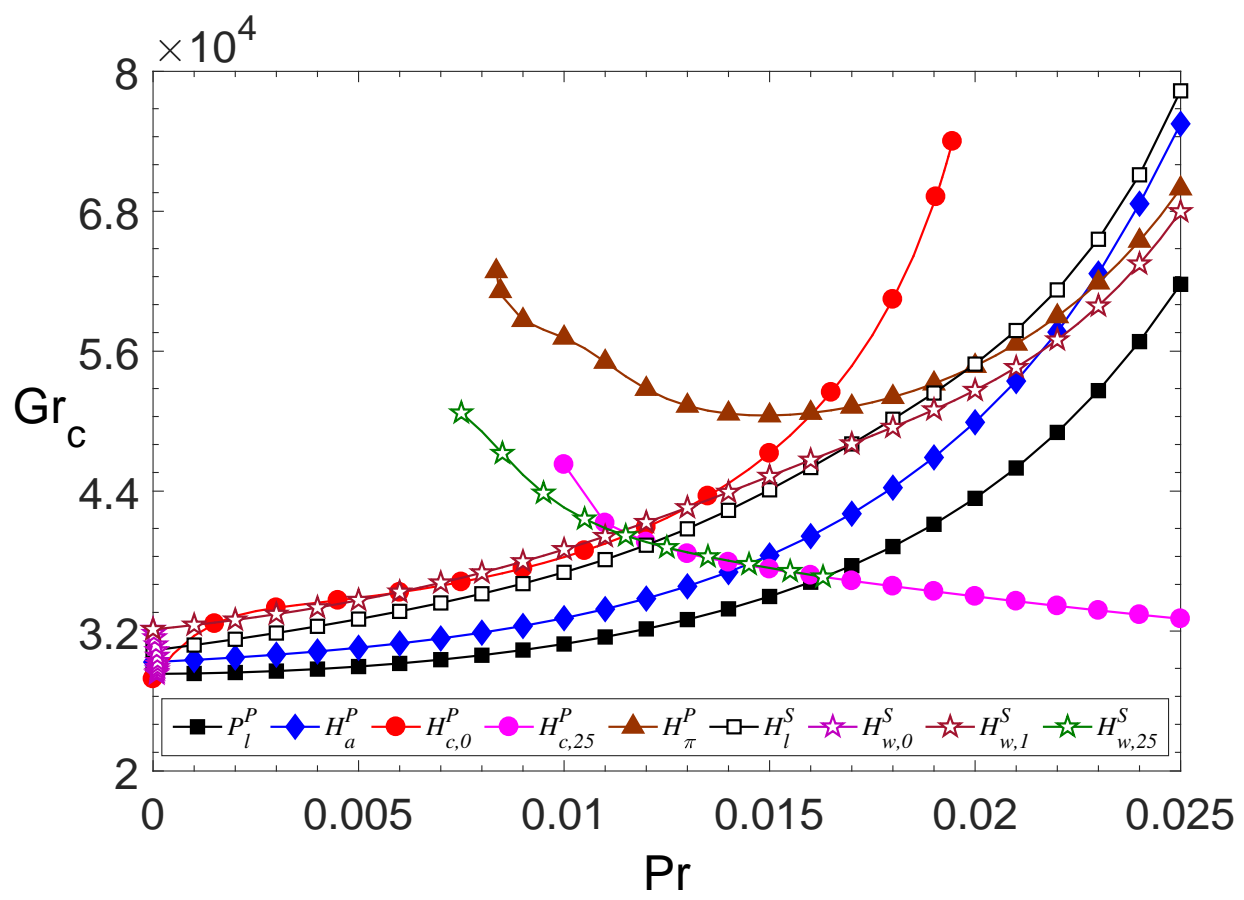

(b)

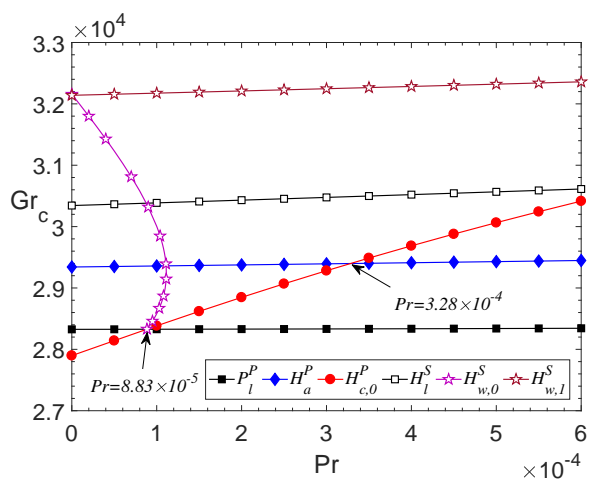

(c)

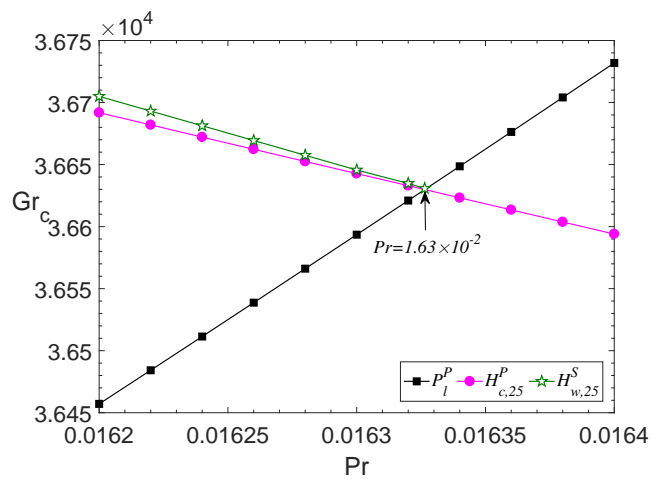

Figure 4: Tracking of the main bifurcation points in the $\left(\operatorname{Pr}, G r_{c}\right)$ parameter space. (a) Global view in the range $0 \leqslant \operatorname{Pr} \leqslant 0.025$. (b) Zoom of the region $\operatorname{Pr} \leqslant 6 \times 10^{-4}$ in order to highlight the crossing of the $P_{l}^{P}$ and $H_{c, 0}^{P}$ primary bifurcation points at $\operatorname{Pr}=8.83 \times 10^{-5}$ with the creation of the $H_{w, 0}^{S}$ secondary bifurcation at this co-dimension 2 point. (c) Zoom around the crossing of the $P_{l}^{P}$ and $H_{c, 25}^{P}$ primary bifurcation points at $\mathrm{Pr}=1.63 \times 10^{-2}$ highlighting the creation of the $H_{w, 25}^{S}$ secondary bifurcation at this other co-dimension 2 point. In this figure, squares are used for modes with $S_{l}$ (left-right) symmetry, circles for modes with $S_{c}$ (center) symmetry, triangles for modes with $S_{\pi}$ ( $\pi$-rotational) symmetry, diamonds for modes with all the symmetries, and stars for modes without symmetry. The symbols are filled (empty) for primary (secondary) bifurcation points. The thresholds for $P_{l}^{P}$, the only steady bifurcation, are given as black squares. 
(a)

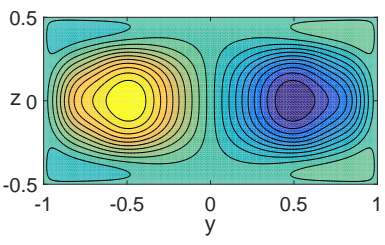

(d)

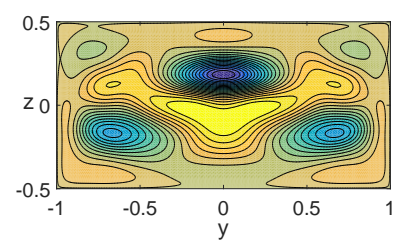

(b)

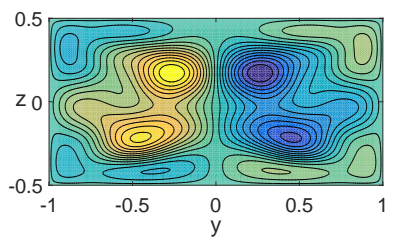

(e)

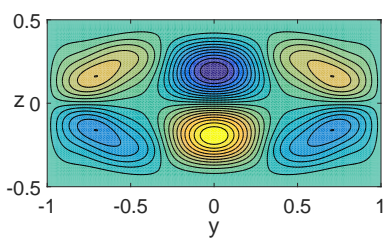

(c)

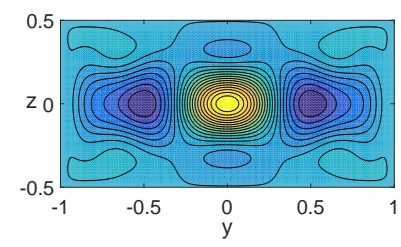

(f)

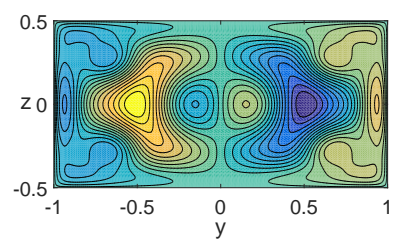

Figure 5: Contours of the longitudinal velocity perturbation $u^{\prime}(y, z)$ in the transverse $V_{t}$ plane for the critical modes (real part) associated with different bifurcation points. The sub-figures (a)-(f) correspond to $H_{c, 25}^{P}$ at $P r=0.025, H_{w, 25}^{S}$ at $P r=0.015, P_{l}^{P}, H_{l}^{S}$ and $H_{a}^{P}$ at $\operatorname{Pr}=0.005$ and finally $H_{c, 0}^{P}$ at $\operatorname{Pr}=0$, respectively.

in Fig. 4 and the plots of the corresponding critical modes in the transverse $V_{t}$ plane are given in Fig. 5. Note that, in some cases, we have introduced an index in the subscript in order to distinguish bifurcation points corresponding to modes with the same symmetry. The index 0 was chosen for the bifurcation $H_{c, 0}^{P}$ which is the first to appear at $\mathrm{Pr}=0$ and for the bifurcation $H_{w, 0}^{S}$ issued from $H_{c, 0}^{P}$. Similarly the index 25 was chosen for the bifurcation $H_{c, 25}^{P}$ which is the first to appear at $\operatorname{Pr}=25 \times 10^{-3}$ and for the bifurcation $H_{w, 25}^{S}$ issued from $H_{c, 25}^{P}$. Finally the index 1 was chosen for the other Hopf bifurcation point without symmetry $H_{w, 1}^{S}$.

Based on the crossings of the first bifurcation points on the primary branch observed in Fig. 4, three main regions can be identified: for $\operatorname{Pr} \leqslant 8.83 \times 10^{-5}$, the Hopf mode $H_{c, 0}^{P}$ (Fig. 5f) destabilises the primary branch and gives birth to a limit cycle with the $S_{c}$ symmetry and small frequency $\left(f_{c} \approx 2.70\right.$ at $\left.\operatorname{Pr}=0\right)$. For larger values of the Prandtl number $\left(8.83 \times 10^{-5} \leqslant \operatorname{Pr} \leqslant 1.63 \times 10^{-2}\right)$, the primary branch is first destabilized by the steady mode $P_{l}^{P}$ (Fig. $5 \mathrm{c}$ ), which will trigger a stable steady solution with the $S_{l}$ symmetry. Finally, for $1.63 \times 10^{-2} \leqslant P r \leqslant 2.5 \times 10^{-2}$, it is the Hopf mode $H_{c, 25}^{P}$ (Fig. 5a) that destabilizes the primary branch. A limit cycle with the $S_{c}$ symmetry is also generated at this point, but it has a larger frequency $\left(f_{c} \approx 13.8\right.$ at $\left.\operatorname{Pr}=0.025\right)$ than for very small $\operatorname{Pr}$ values. Note that the intersection points located at $\operatorname{Pr}=8.83 \times 10^{-5}$ and $\operatorname{Pr}=1.63 \times 10^{-2}$ are pitchfork-Hopf codimension-2 bifurcations. They are at the origin of the creation of two Hopf bifurcation points on the secondary branch, $H_{w, 0}^{S}$ (from $H_{c, 0}^{P}$ and $P_{l}^{P}$ ) and $H_{w, 25}^{S}$ (from $H_{c, 25}^{P}$ and $P_{l}^{P}$ ), respectively (see Figs. $4 \mathrm{~b}$ and $4 \mathrm{c}$ ). We will see later that the codimension-2 point at $\operatorname{Pr}=1.63 \times 10^{-2}$ is also at the origin of the creation of a pitchfork of cycles on the branch of periodic solutions that emerges from $H_{c, 25}^{P}$. The point $H_{w, 25}^{S}$ evolves then towards smaller Prandtl numbers with an increasing threshold 
whereas $H_{w, 0}^{S}$ has also an increasing threshold but evolves slightly towards higher Prandtl numbers before turning at $\operatorname{Pr} \approx 1.1 \times 10^{-4}$ and evolving down to $\operatorname{Pr}=0$. This latter behaviour implies that in the range $8.83 \times 10^{-5} \leqslant P r \leqslant 1.1 \times 10^{-4}$, the secondary steady branch is successively destabilized and then re-stabilized by $H_{w, 0}^{S}$, before being destabilized by $H_{l}^{S}$.

In addition, when the first bifurcation is steady $\left(8.83 \times 10^{-5} \leqslant \operatorname{Pr} \leqslant 1.63 \times 10^{-2}\right)$, the bifurcated secondary branch is destabilized either by the Hopf mode $H_{l}^{S}$ (Fig. $5 \mathrm{~d}$ ) giving a limit cycle which keeps the $S_{l}$ symmetry of the branch $\left(\operatorname{Pr} \leqslant 1.21 \times 10^{-2}\right)$ or by the Hopf mode $H_{w, 25}^{S}$ (Fig. 5b) that breaks this $S_{l}$ symmetry and initiates a cycle without symmetries $\left(\mathrm{Pr} \geqslant 1.21 \times 10^{-2}\right)$. Finally two other crossings of bifurcation points occurring on the primary branch can influence the dynamics of transitions in the cavity in the intermediate range of $\mathrm{Pr}$ : the crossing between the Hopf bifurcation points $H_{c, 0}^{P}$ and $H_{a}^{P}$ at very low $\operatorname{Pr}\left(\operatorname{Pr}=3.28 \times 10^{-4}\right)$ and the crossing between the Hopf bifurcation points $H_{a}^{P}$ and $H_{c, 25}^{P}$ at $P r=1.44 \times 10^{-2}$. The values of $\operatorname{Pr}$ given for all these crossings of bifurcation points have been obtained with a good precision by a Newton method.

The bifurcation analysis of the steady flows presented in this section has shown that only two steady flow solutions exist for the cavity considered here and in the range of $\operatorname{Pr}$ studied. There are however many different Hopf bifurcation points on these steady branches, which can generate different oscillatory flow solutions. The study of these oscillatory flows will be presented in the next section.

\section{Bifurcation analysis of the oscillatory flows}

The bifurcation analysis of the oscillatory flows has been performed thanks to the method presented in section 2 allowing the continuation of cycles and the calculation of their stability. In what follows, we first present the results for different Prandtl numbers, for $\operatorname{Pr}=0.025$ and $\operatorname{Pr}=0$ in the regions where Hopf bifurcation points first destabilize the primary branch and for $\operatorname{Pr}=0.015$ and $\operatorname{Pr}=0.005$ in the region where exists a stable steady secondary branch. We will then give a more global view of all the transitions encountered in the whole $\operatorname{Pr}$ range. We begin with the simplest case at $\operatorname{Pr}=0.025$ and then consider the cases at successively smaller values of $\operatorname{Pr}$. Note that the cycles will be very often called according to the symmetry they keep, i.e. $S_{l}, S_{\pi}$ or $S_{c}$ cycles. $S_{a}$ and $S_{w}$ cycles will also be used for cycles with all the symmetries and cycles without symmetry, respectively. The cycles will be also plotted with specific colors: green for the $S_{c}$ cycles, red for the $S_{a}$ cycles, blue for the $S_{l}$ cycles, and pink for the $S_{w}$ cycles. Finally, as already mentioned, the bifurcations appearing on the cycles will be denoted as $P$ (pitchfork) or $H$ (generalized Hopf) with a superscript giving information on the cycle $\left(C_{l}\right.$ for a cycle with $S_{l}$ symmetry, for example) and a subscript indicating the symmetry kept by the critical mode.

\section{1. $\operatorname{Pr}=0.025$}

As shown in Table 2 and in Fig. 4a, the flow for $\operatorname{Pr}=0.025$ is a stable steady convective loop (solution on the primary branch with all the symmetries) up to its destabilization by the $H_{c, 25}^{P}$ mode at $G r_{c}=33055$. The destabilizing mode only keeps the $S_{c}$ symmetry (Fig. 5a) and is associated with a critical frequency equal to 13.8. At the critical point, the flow is found to undergo a supercritical Hopf bifurcation toward a stable limit cycle (Fig. $6 a)$. The resulting structure is thus an oscillatory flow which keeps the central symmetry. This $S_{c}$ cycle has a growing amplitude with the increase of $G r$ and remains stable in a large range of $G r$. This could be connected to the absence of bifurcation points in the 
(a)

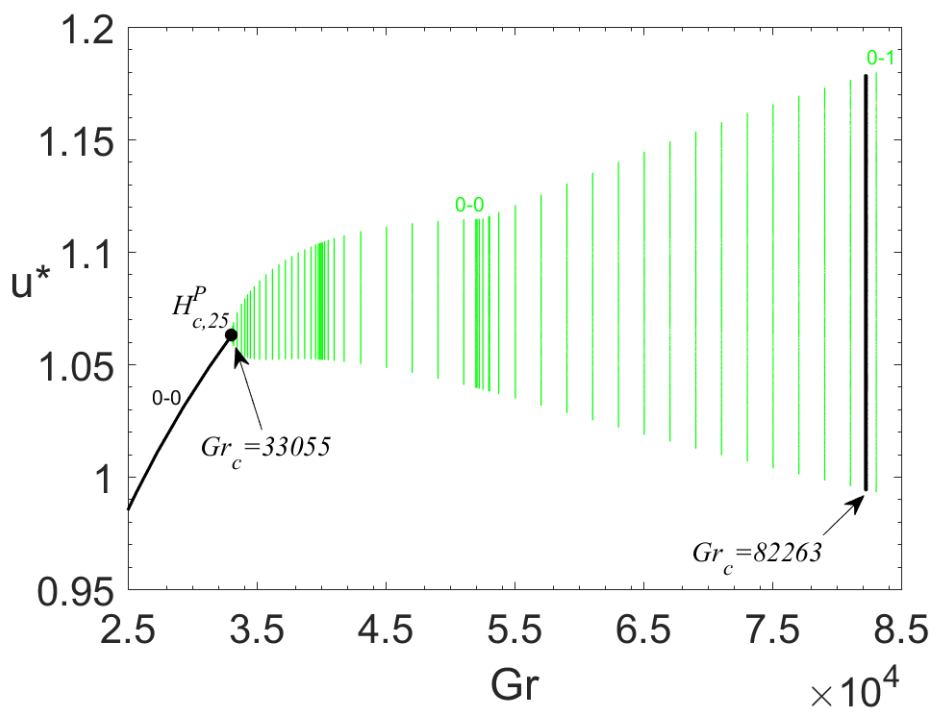

(b)

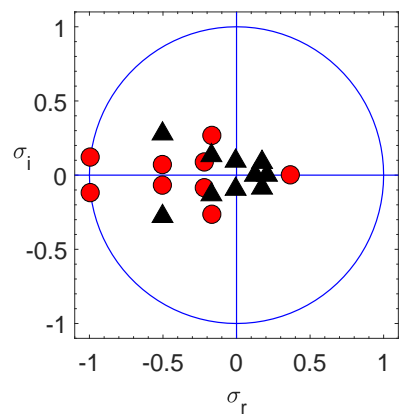

Figure 6: Convective solutions for $\operatorname{Pr}=0.025$ : (a) Bifurcation diagram ( $u^{*}$ versus $G r$ ) showing the stable steady solution with all the symmetries (primary branch) and the limit cycle with $S_{c}$ symmetry initiated at the Hopf point $H_{c, 25}^{P}\left(G r_{c}=33055\right)$ on the primary branch and destabilized at $G r_{c}=82263$. The stability of the different states is indicated by the number of unstable eigenvalues $n-m$ (see text in section 4); (b) Floquet multipliers associated with the limit cycle for $G r=52000$ (black triangles) and for $G r=82263$ (red circles), showing that the limit cycle is destabilized by a pair of complex conjugate eigenvalues.

vicinity of the destabilizing Hopf point $H_{c, 25}^{P}$ (the next bifurcation $P_{l}^{P}$ occurs only at $G r_{c}=61737$ ) (see Table 2 and Fig. 4). To illustrate the stability of the cycle, we give the Floquet multipliers of largest norm for two values of the Grashof number in Fig. 6b. For $G r=52000$ (black triangles), all the dominant Floquet multipliers are inside the unit circle, which means that the limit cycle is stable. In contrast, when the Grashof number is increased up to $G r=82263$ (red circles), a pair of complex conjugate Floquet multipliers cross the unit circle, indicating the destabilization of the limit cycle at a Naimark-Sacker 
bifurcation, with an eigenvector keeping the $S_{c}$ symmetry. This bifurcation is supercritical and thus gives birth to a stable quasi-periodic flow with central symmetry ( $\mathrm{QP}_{c, 2}$ state) beyond $G r_{c}=82263$. Some numerical simulations obtained by time stepping confirm this transition: indeed, if the solution is still oscillatory periodic for $G r=80000$, it becomes quasi-periodic for $G r=85000$.

\section{2. $\operatorname{Pr}=0.015$}

The bifurcation diagram corresponding to $P r=0.015$ is given in Fig. 7a. This diagram shows a different route toward quasi-periodicity. Two steady solutions (solid black lines) and two periodic solutions (pink and green colors) are involved, with connections between these solutions. A third cycle, initiated at the Hopf bifurcation point $H_{a}^{P}\left(G r_{c}=38482\right)$ on the primary branch and with all the symmetries (red color), also exists in the same $G r$ range, but remains unstable and will not participate to the observed dynamics of the flow at this value of $\mathrm{Pr}$.

We can describe more precisely the dynamics of the flow in this case. The basic steady flow with all the symmetries (primary branch) is stable up to the bifurcation point $P_{l}^{P}$ at $G r_{c}=34966$ where a supercritical pitchfork bifurcation occurs leading to the stable steady flow with $S_{l}$ symmetry (secondary branch). This secondary branch is then destabilized at the Hopf bifurcation point $H_{w, 25}^{S}\left(G r_{c}=37413\right)$. The marginal mode at this point (Fig. $5 \mathrm{~b}$ ) is antisymmetric with respect to the $S_{l}$ symmetry of the branch, so that the resulting flow on the emerging limit cycle has no more symmetry. This $S_{w}$ cycle (pink color) evolves super-critically and is then stable (Fig. 7c). It eventually disappears at $G r_{c}=38588$, a pitchfork bifurcation of cycles $P_{w}^{C_{c, 25}}$ on the $S_{c}$ cycle branch (green color). There are then in fact two equivalent branches of $S_{w}$ cycles, which emerge from the two equivalent steady secondary branches initiated at $P_{l}^{P}$ and mentioned in section 4 and disappear at $P_{w}^{C_{c, 25}}$.

On the other hand, this $S_{c}$ cycle (green color) is initiated at the Hopf bifurcation point $H_{c, 25}^{P}\left(G r_{c}=37314\right)$ on the primary branch, beyond the first steady bifurcation at $P_{l}^{P}$. The cycle is first unstable, as it bifurcates from an already unstable steady branch. When the Grashof number is increased, the cycle is stabilized at the $P_{w}^{C_{c, 25}}$ pitchfork bifurcation point, when one real Floquet multiplier crosses the unit circle (Fig. 7b, green squares). For further increase of $G r$, the $S_{c}$ cycle evolves with a regularly increasing amplitude until its destabilization at a supercritical Naimark-Sacker bifurcation. The pair of complex conjugate Floquet multipliers destabilizing the cycle is shown in Fig. 7b (red circles). These Floquet multipliers are associated with an eigenvector keeping the central symmetry. Beyond this bifurcation at $G r_{c}=46209$, a stable quasi-periodic state with $S_{c}$ symmetry $\left(\mathrm{QP}_{c, 2}\right.$ state) can then be observed.

To summarize, for $\operatorname{Pr}=0.015$ and $G r \leqslant 47000$, we observe a sequence of different solutions which are stable on successive ranges of $G r$ : the steady solution with all the symmetries on the primary branch for $G r \leqslant 34965$, the steady solution with $S_{l}$ symmetry on the secondary branch for $34965 \leqslant G r \leqslant 37413$, the limit cycle with no-symmetry for $37413 \leqslant G r \leqslant 38588$, the limit cycle with $S_{c}$ symmetry for $38588 \leqslant G r \leqslant 46209$, and finally the quasi-periodic solution with $S_{c}$ symmetry for $G r \geqslant 46209$.

$$
\text { 5.3. } \operatorname{Pr}=0.005
$$

The convective situation at $\operatorname{Pr}=0.005$ still differs from the previous situations studied, as a coexistence of stable steady and oscillatory solutions will be observed.

The bifurcation diagram giving the different convective solutions for $\operatorname{Pr}=0.005$ is shown in Fig. 8a. When the Grashof number is increased, as for $\operatorname{Pr}=0.015$, we first 
(a)

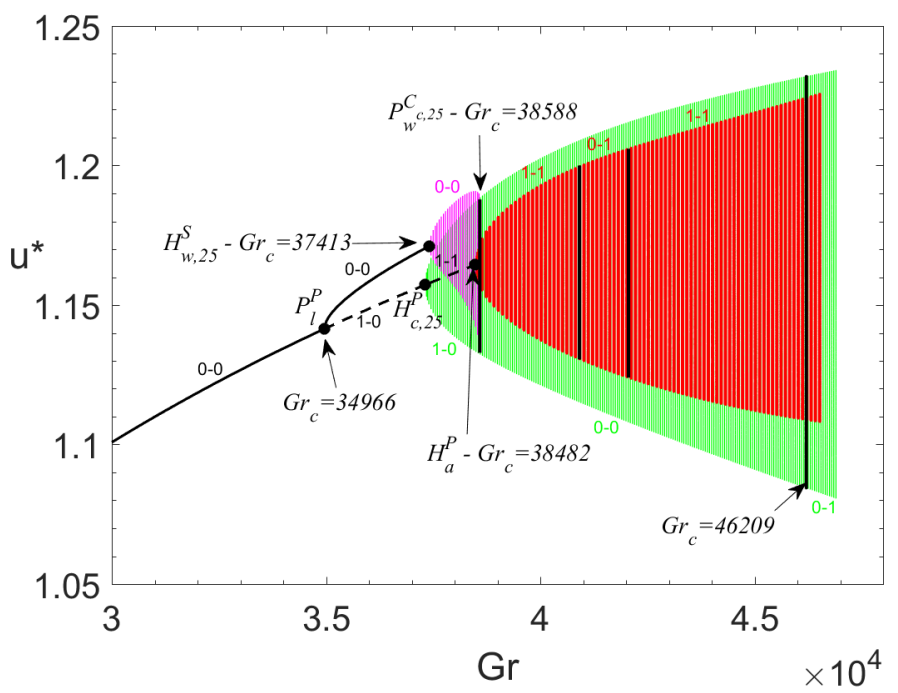

(b)

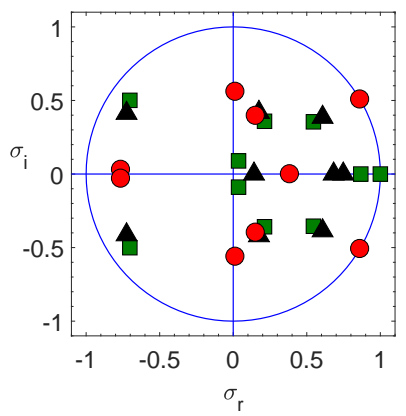

(c)

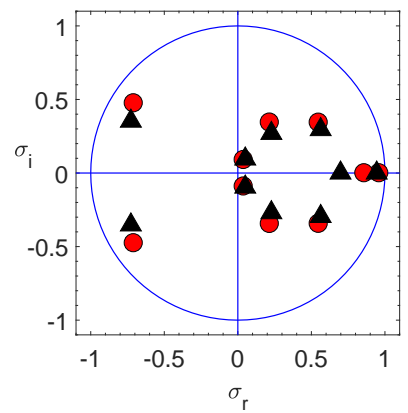

Figure 7: Convective solutions for $\operatorname{Pr}=0.015$ : (a) Bifurcation diagram ( $u^{*}$ versus $G r$ ) showing the stable steady solutions on the primary and secondary branches (solid black lines), the stable cycle without symmetries (pink color), which stabilizes the cycle with $S_{c}$ symmetry (green color) at $P_{w}^{C_{c, 25}}$ at $G r_{c}=38588$ and up to $G r_{c}=46209$, and the cycle with all the symmetries (red color), which remains unstable. The stability of the different states is indicated by the number of unstable eigenvalues $n-m$ (see text in section 4); (b) Floquet multipliers associated with the limit cycle initiated at the Hopf point $H_{c, 25}^{P}$ for $G r=38588$ (green squares), $G r=40000$ (black triangles) and $G r=46209$ (red circles); (c) Floquet multipliers associated with the limit cycle initiated at the Hopf point $H_{w, 25}^{S}$ for $G r_{c}=38000$ (black triangles) and $G r=38490$ (red circles).

observe the two steady flow solutions, the solution with all the symmetries on the primary branch, stable up to the pitchfork bifurcation $P_{l}^{P}$ at $G r_{c}=28957$, and the steady solution with $S_{l}$ symmetry on the bifurcated secondary branch. This secondary branch is stable up to the Hopf bifurcation $H_{l}^{S}$ at $G r_{c}=33026$. This Hopf bifurcation keeps the $S_{l}$ symmetry of the secondary branch (see the marginal mode in Fig. 5d), but the limit 
(a)

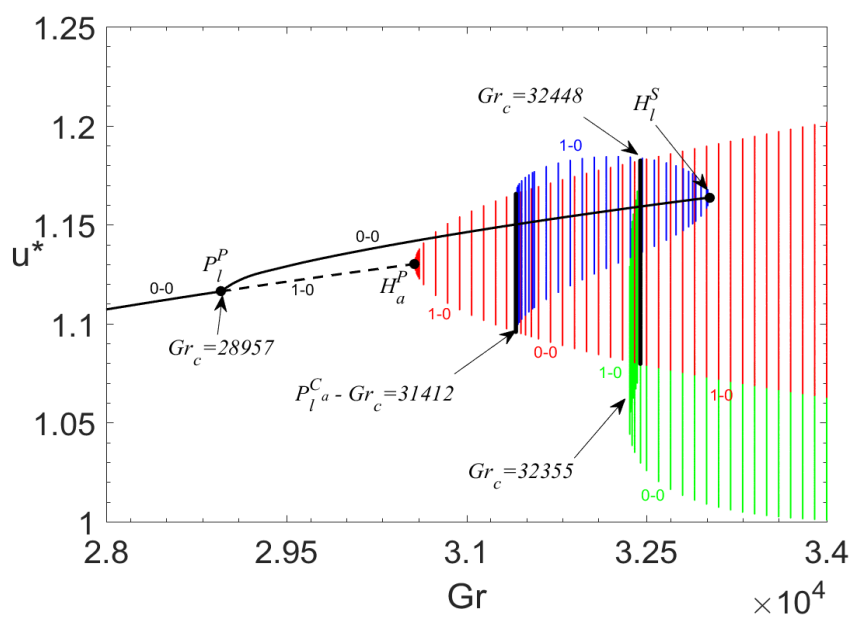

(b)

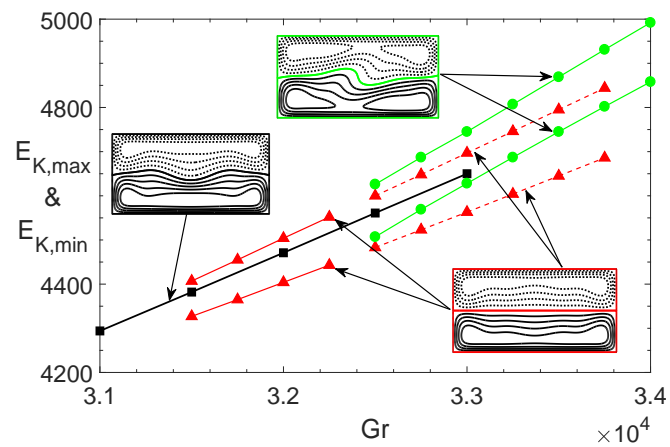

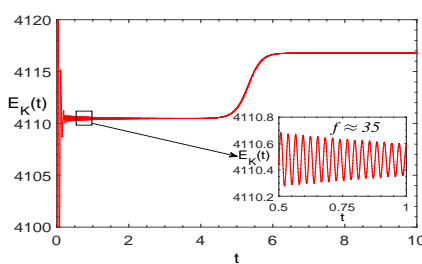

(c) $G r=30000$

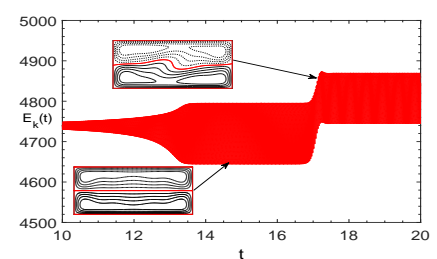

(d) $G r=33500$

Figure 8: Convective solutions for $\operatorname{Pr}=0.005$ : (a) Bifurcation diagram ( $u^{*}$ versus $G r$ ) showing the stable steady solutions on the primary and secondary branches (solid black lines), the unstable subcritical cycle with $S_{l}$ symmetry (blue color), the cycle with all the symmetries (red color) stabilized at the pitchfork bifurcation $P_{l}^{C_{a}}$ at $G r_{c}=31412$ and up to $G r_{c}=32448$, and the cycle with $S_{c}$ symmetry (green color) stable above the saddlenode point at $G r_{c}=32355$. The stability of the different states is indicated by the number of unstable eigenvalues $n-m$ (see text in section 4); (b) Bifurcation diagram obtained by time-stepping simulations giving the averaged kinetic energy maximum $E_{K, \max }$ and minimum $E_{K, \text { min }}$ during a cycle for the different solutions obtained: steady solutions on the secondary branch (black squares), periodic solutions with $S_{c}$ symmetry (green circles) and with all symmetries (red diamonds, a dashed line is used for transient states); (c) and (d) Time series $E_{K}(t)$ for $G r=30000$ and 33500, respectively. Insets give the longitudinal velocity in the transverse $V_{t}$ plane 


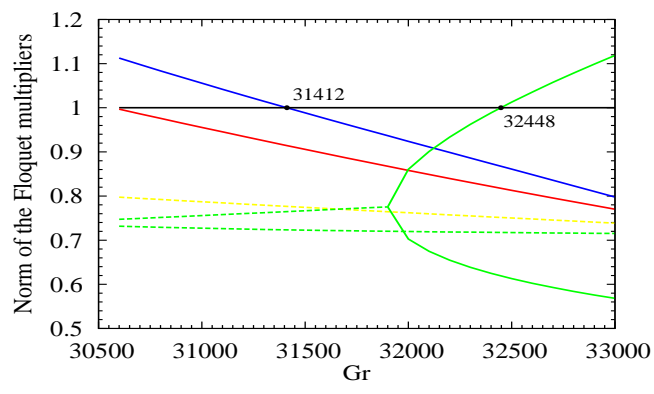

(a)

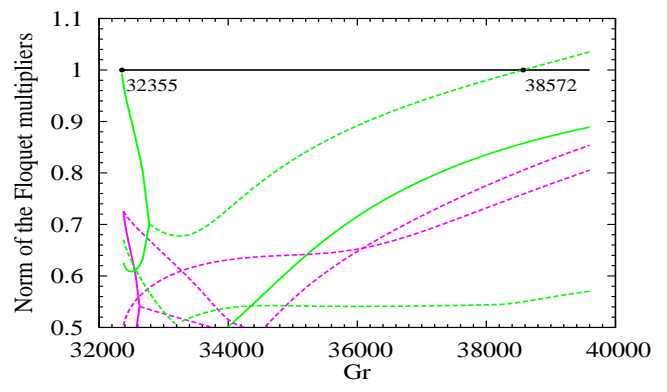

(b)

Figure 9: Variation of the spectrum (norm of the main Floquet multipliers) with $G r$ for (a) the limit cycle with all the symmetries and (b) the limit cycle with the $S_{c}$ symmetry. Solid (dashed) lines are used for steady (complex conjugate) Floquet multipliers. The associated modes have all the symmetries (red line), the $S_{l}$ symmetry (blue line), the $S_{c}$ symmetry (green line), the $S_{\pi}$ symmetry (yellow line) or no symmetry (pink line). The results are for $\operatorname{Pr}=0.005$.

cycle with $S_{l}$ symmetry that is created (blue color) evolves sub-critically and is then unstable at onset. This $S_{l}$ cycle remains unstable while developing for decreasing $G r$ and eventually disappears at $G r_{c}=31412$, a pitchfork bifurcation of cycles $P_{l}^{C_{a}}$ on the $S_{a}$ cycle branch (red color). Here also, there are two equivalent branches for this $S_{l}$ cycle, which emerge from the two equivalent steady secondary branches initiated at $P_{l}^{P}$ and disappear at $P_{l}^{C_{a}}$.

On the other hand, the $S_{a}$ cycle with all the symmetries (red color), initiated at the Hopf point $H_{a}^{P}\left(G r_{c}=30569\right)$ along the primary steady branch, beyond the steady bifurcation $P_{l}^{P}$, evolves super-critically, as shown in Fig. 8a. The stability changes for this cycle can be obtained from the variation with $G r$ of the norm of the main Floquet multipliers plotted in Fig. 9a. This limit cycle is unstable at onset, with a real positive Floquet multiplier of norm greater than one (solid blue line in Fig. 9a), because it is initiated on an already unstable steady branch. The norm of this Floquet multiplier decreases linearly as $G r$ is increased and becomes less than 1 (crossing of the unit circle) at $G r_{c}=31412$, i.e. at the pitchfork bifurcation point $P_{l}^{C_{a}}$. The $S_{a}$ cycle, stable beyond this point, develops in amplitude (Fig. 8a), before being destabilized at $G r_{c}=32448$ by a steady eigenvector (solid green curve in Fig. 9a), which only keeps the central symmetry. The cycle initiated at this point holds thus the $S_{c}$ symmetry. It is first unstable as it bifurcates sub-critically from the $S_{a}$ cycle, but it is soon stabilized by a saddle-node point at $G r_{c} \approx 32355$ and then evolves for increasing Grashof numbers. The norms of the main Floquet multipliers corresponding to this $S_{c}$ cycle are given in Fig. 9b. We see the stabilisation of the cycle at $G r_{c}=32355$ (solid green line) and its destabilization by a pair of complex conjugate Floquet multipliers (dashed green line) at $G r_{c}=38572$. A quasi-periodic flow $\left(\mathrm{QP}_{c, 2}\right.$ state), still with $S_{c}$ symmetry, appears for larger Grashof numbers beyond this supercritical Naimark-Sacker bifurcation. Note that the dynamics observed for the $S_{a}$ cycle, with the interaction with the $S_{l}$ cycle and then with a $S_{c}$ cycle, was already present for $\operatorname{Pr}=0.015$. It was not detailed, however, for $\operatorname{Pr}=0.015$ because it did not lead to stable solutions in this case.

These results have shown the coexistence of different stable solutions in the same $G r$ range: coexistence of the steady solution with $S_{l}$ symmetry with the $S_{a}$ cycle for $31412 \leqslant$ $G r \leqslant 32448$ and with the $S_{c}$ cycle for $32355 \leqslant G r \leqslant 33026$, and even coexistence of the 
three solutions for $32355 \leqslant G r \leqslant 32448$. In order to highlight this complex dynamics for $\operatorname{Pr}=0.005$, some additional time-stepping numerical simulations were carried out for different Grashof numbers ranging from 30000 to 35000 . The results are first presented in Figs. 8 c- 8 d in terms of the time variation of $E_{K}(t)$ for $G r=30000$ and 33500 , respectively. An important observation is that the behaviour of the states obtained by time-stepping simulations is influenced by the different solutions and instability modes present in the vicinity of the considered Grashof number. For instance, for $G r=30000$ (Fig. 8c), there exist the primary steady solution branch, for which the main instability modes are the already unstable steady $P_{l}^{P}$ mode and the still stable oscillatory $H_{a}^{P}$ mode with the frequency $f\left(H_{a}^{P}\right) \approx 35$, and the stable secondary steady branch that has bifurcated at $G r_{c}\left(P_{l}^{P}\right)=28957$ (see Fig. 8a). We naturally expect that the solution at long times will be the stable steady state with $S_{l}$ symmetry on the secondary branch. In practice, we notice the existence of damped oscillations connected with the $H_{a}^{P}$ mode (their frequency is close to $f\left(H_{a}^{P}\right)$ ) which lead to a transient convergence towards the unstable steady state on the primary branch, and it is only for $t \approx 5$ that the stable steady state on the secondary branch is eventually reached. A similar behaviour is also observed for $G r=33500$ (Fig. 8d): a transient convergence towards the $S_{a}$ cycle (unstable state) is observed, before the emergence of the $S_{c}$ cycle (stable state) for $t>17$. Note that such a behaviour was already observed during transient calculations in two dimensional differentially heated cavities by Pulicani et al. (1990). The different solutions reached during the time-stepping numerical simulations are summarized in the bifurcation diagram given in Fig. $8 \mathrm{~b}$. The solutions are plotted as $E_{K, \max }$ and $E_{K, \text { min }}$, maximum and minimum values of $E_{K}(t)$ during a cycle, respectively $\left(E_{K, \max }=E_{K, \min }\right.$ for a steady solution). The green circles represent the $S_{c}$ cycle, the black squares indicate the steady flow with the $S_{l}$ symmetry on the secondary branch and finally, red diamonds are used for the $S_{a}$ cycle. For these last solutions, the cycles obtained as transient states are given as dashed lines. We observe that this bifurcation diagram obtained by time-stepping simulations is coherent with that obtained by continuation in Fig. 8a. Note, however, that the subcritical cycle with $S_{l}$ symmetry is never observed as a transient cycle during the time-stepping simulations: only damped oscillations towards the stable steady state with $S_{l}$ symmetry are generally obtained. This could be due to the fact that, in this case, both the subcritical cycle and the stable steady state have the same symmetries.

\section{4. $\operatorname{Pr}=0$}

The case $\operatorname{Pr}=0$ has long been considered as an interesting approximation to simulate the flows of liquid metals with small $\mathrm{Pr}$, such as those observed in crystal growth processes. The temperature field, in this limit case, is purely diffusive, with a linear variation of the temperature between the two imposed values at the endwalls, and does not need to be solved.

The specificity in this case is that the first instability of the steady basic flow on the primary branch occurs at the Hopf bifurcation $H_{c, 0}^{P}$, which initiates a stable cycle with central symmetry and low frequency $\left(G r_{c}=27893\right.$ and $f_{c}=2.7$, see Fig. $5 \mathrm{f}$ and Tab. 2). This $S_{c}$ cycle (Fig. 10a) is destabilized at the Naimark-Sacker bifurcation $H_{c}^{C_{c, 0}}$ by a mode with the same $S_{c}$ symmetry, creating a quasi-periodic stable solution ( $\mathrm{QP}_{c, 1}$ state). On the same primary branch, we find the usual $P_{l}^{P}$ bifurcation to the steady secondary branch, which is unstable here, and a Hopf bifurcation $H_{a}^{P}$ keeping all the symmetries. At this Hopf point, a $S_{a}$ cycle appears super-critically. It is two times unstable at onset, becomes one time unstable at $P_{l}^{C_{a}}$ by stabilization of a steady mode with $S_{l}$ symmetry and is eventually stabilized at $H_{c}^{C_{a}}$ by stabilization of a complex conjugate mode with 
$S_{c}$ symmetry. It then remains stable up to $G r_{c}=31241$, where a steady mode with $S_{c}$ symmetry is destabilized. At these different critical values along the $S_{a}$ cycle, different new oscillatory solutions will appear. At $P_{l}^{C_{a}}$, an unstable $S_{l}$ cycle appears for larger $G r$ and this cycle eventually disappears at the Hopf point $H_{l}^{S}$ on the secondary steady branch. At $H_{c}^{C_{a}}$, a stable quasi-periodic solution with central symmetry is created for smaller $G r$. This stable $\mathrm{QP}_{c, 1}$ state exists down to the already mentioned bifurcation $H_{c}^{C_{c, 0}}$ on the low frequency $S_{c}$ cycle. Finally, at $G r_{c}=31241$, a stable $S_{c}$ cycle with high frequency appears for larger $G r$. This cycle remains stable up to $G r_{c}=31476$ where a quasi-periodic centro-symmetric solution $\left(\mathrm{QP}_{c, 2}\right.$ state) is created beyond a supercritical Naimark-Sacker bifurcation. The $\mathrm{QP}_{c, 1}$ quasi-periodic state obtained for $\operatorname{Pr}=0$ is illustrated in Fig. 10b by time evolutions of $u^{*}$ : we see the change of this state from a solution with small amplitude short-period oscillations on a long-period signal $\left(G r=29400\right.$, close to $\left.H_{c}^{C_{c, 0}}\right)$ to a solution with short-period oscillations modulated in amplitude $\left(G r=30400\right.$, close to $\left.H_{c}^{C_{a}}\right)$.

\subsection{Global view of the solutions}

A global presentation of the different solutions obtained when $G r$ is increased for the four chosen Prandtl numbers is given in Fig. 11. The solutions are given by horizontal lines covering their domain of existence in terms of $G r$, these lines being solid lines in the range where the solutions are stable. As in the previous figures, different colours are used for the solutions, depending on their symmetries (green for the $S_{c}$ symmetry, red for the $S_{a}$ symmetry, blue for the $S_{l}$ symmetry, and pink for the $S_{w}$ symmetry). For each Prandtl number, the solutions are presented from $G r=27000$ up to values of $G r$ allowing to reach quasi-periodic states, i.e. $G r=34000,39000,47000$ and 83500 for $\operatorname{Pr}=0,0.005,0.015$ and 0.025 , respectively. We can see that many different solutions (steady, periodic, quasiperiodic) with different symmetries can be obtained. The path followed to go from the basic steady solution to the final quasi-periodic state through periodic states is different for each $\operatorname{Pr}$ number studied. In any case, however, it was found that the last periodic solution is a $S_{c}$ cycle, which is then destabilized at a Naimark-Sacker bifurcation setting up a quasi-periodic state with the same $S_{c}$ symmetry.

\section{Change of the transition scenarios with $\mathrm{Pr}$}

Until now, we have presented some bifurcation analyses for different Prandtl numbers, but without connecting these cases between them. The idea is now to use these few examples to deepen our understanding of how the transition scenarios between the different observed stable solutions evolve when changing the Prandtl number.

The results presented in section 5 have shown that many Hopf bifurcation points, on the primary as well as on the secondary steady branch, are involved in the transition scenarios. There are also important connections between Hopf bifurcation points on the secondary branch and Hopf bifurcation points on the primary branch through interaction of cycles at pitchfork bifurcations: for example, $H_{l}^{S}$ is connected with $H_{a}^{P}$ through the interaction of the $S_{l}$ and $S_{a}$ cycles at $P_{l}^{C_{a}}$ and $H_{w, 25}^{S}$ is connected with $H_{c, 25}^{P}$ through the interaction of the $S_{w}$ and $S_{c}$ cycles at $P_{w}^{C_{c, 25}}$. Finally the transition scenarios appear to be strongly affected by the relative positions of the bifurcation points on the primary branch, i.e. $P_{l}^{P}, H_{a}^{P}, H_{c, 0}^{P}$ and $H_{c, 25}^{P}$, and of the bifurcation points on the secondary branch, i.e. $H_{l}^{S}, H_{w, 0}^{S}$ and $H_{w, 25}^{S}$. The intersection of the paths of these different bifurcation points then plays an important role. All these intersections, already mentioned in section 4, are summarized in Table 3, together with the $\operatorname{Pr}$ ranges which are thus defined. 
(a)

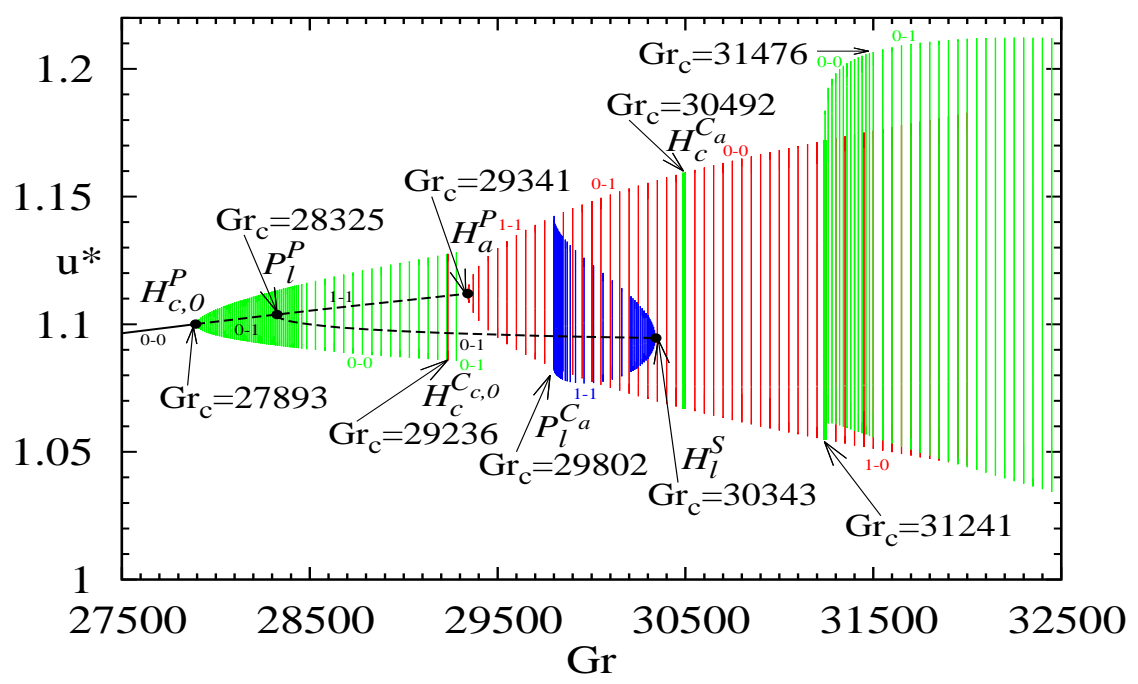

(b)

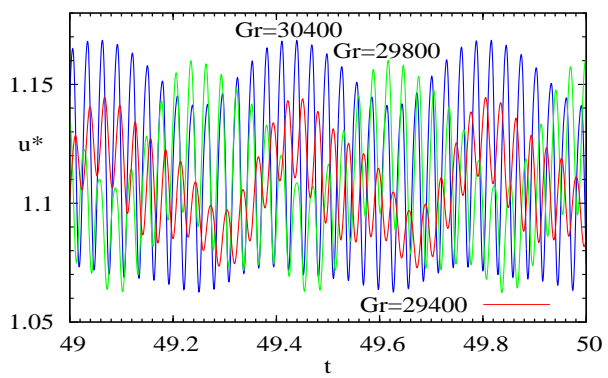

Figure 10: Convective solutions for $\operatorname{Pr}=0$ : (a) Bifurcation diagram ( $u^{*}$ versus $G r$ ) showing the stable steady solution on the primary branch (solid black line), the $S_{c}$ cycle with low frequency (green color) stable from $H_{c, 0}^{P}$ to $H_{c}^{C_{c, 0}}\left(G r_{c}=29236\right)$, the $S_{a}$ cycle (red color) stabilized at $H_{c}^{C_{a}}\left(G r_{c}=30492\right)$ and up to $G r_{c}=31241$, the unstable $S_{l}$ cycle (blue color), and another $S_{c}$ cycle (green color) stable from $G r_{c}=31241$ to $G r_{c}=31476$. Quasi-periodic solutions exist between $H_{c}^{C_{c, 0}}$ and $H_{c}^{C_{a}}\left(\mathrm{QP}_{c, 1}\right.$ state) and above $G r_{c}=31476\left(\mathrm{QP}_{c, 2}\right.$ state). The stability of the different states is indicated by the number of unstable eigenvalues $n-m$ (see text in section 4); (b) Time series $u^{*}(t)$ for the $\mathrm{QP}_{c, 1}$ state $(G r=29400$, red curve; $G r=29800$, green curve; $G r=30400$, blue curve).

For $1.63 \times 10^{-2} \leqslant \operatorname{Pr} \leqslant 2.5 \times 10^{-2}$, in the R6 range, the first bifurcation point is $H_{c, 25}^{P}$. We can then expect that in this range of $\mathrm{Pr}$, as for $\mathrm{Pr}=0.025, H_{c, 25}^{P}$ will give an $S_{c}$ cycle which will evolve with the Grashof number and undergo a Naimark-Sacker bifurcation allowing to quasi-periodicity to set up.

For $1.44 \times 10^{-2} \leqslant P r \leqslant 1.63 \times 10^{-2}$, in the $\mathrm{R} 5$ range, the first bifurcation on the primary branch is now steady at $P_{l}^{P}$ due to the intersection of the $H_{c, 25}^{P}$ and $P_{l}^{P}$ paths. This codimension-2 point leads also to the creation of the Hopf bifurcation $H_{w, 25}^{S}$ on the 

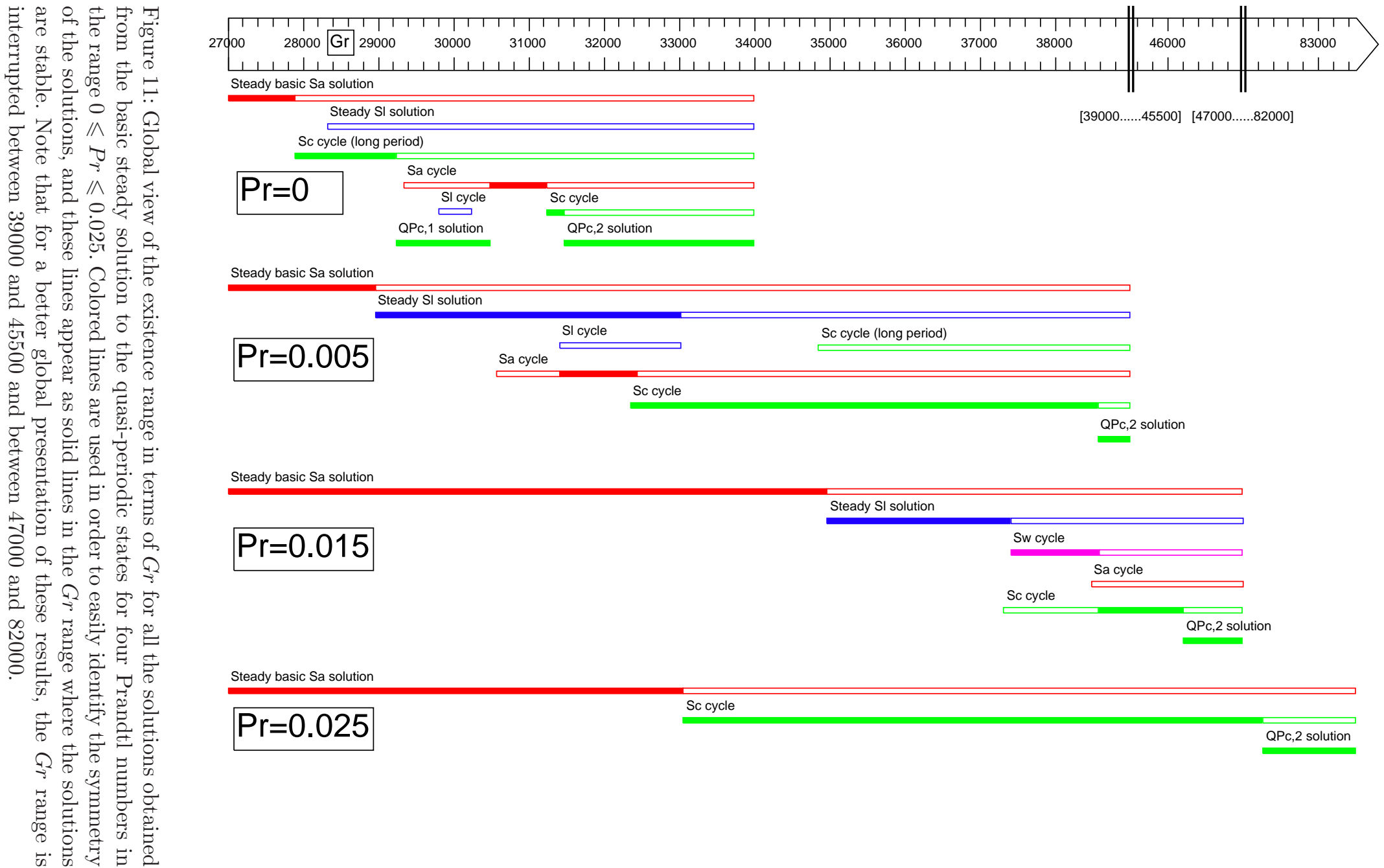

$\mathrm{Pr}=0 \quad \stackrel{\substack{\text { Sl cycle } \\ \text { QPc,1 solution }}}{\frac{\text { Sc cycle }}{\mathrm{QPc}, 2 \text { solution }}}$

Steady basic Sa solution

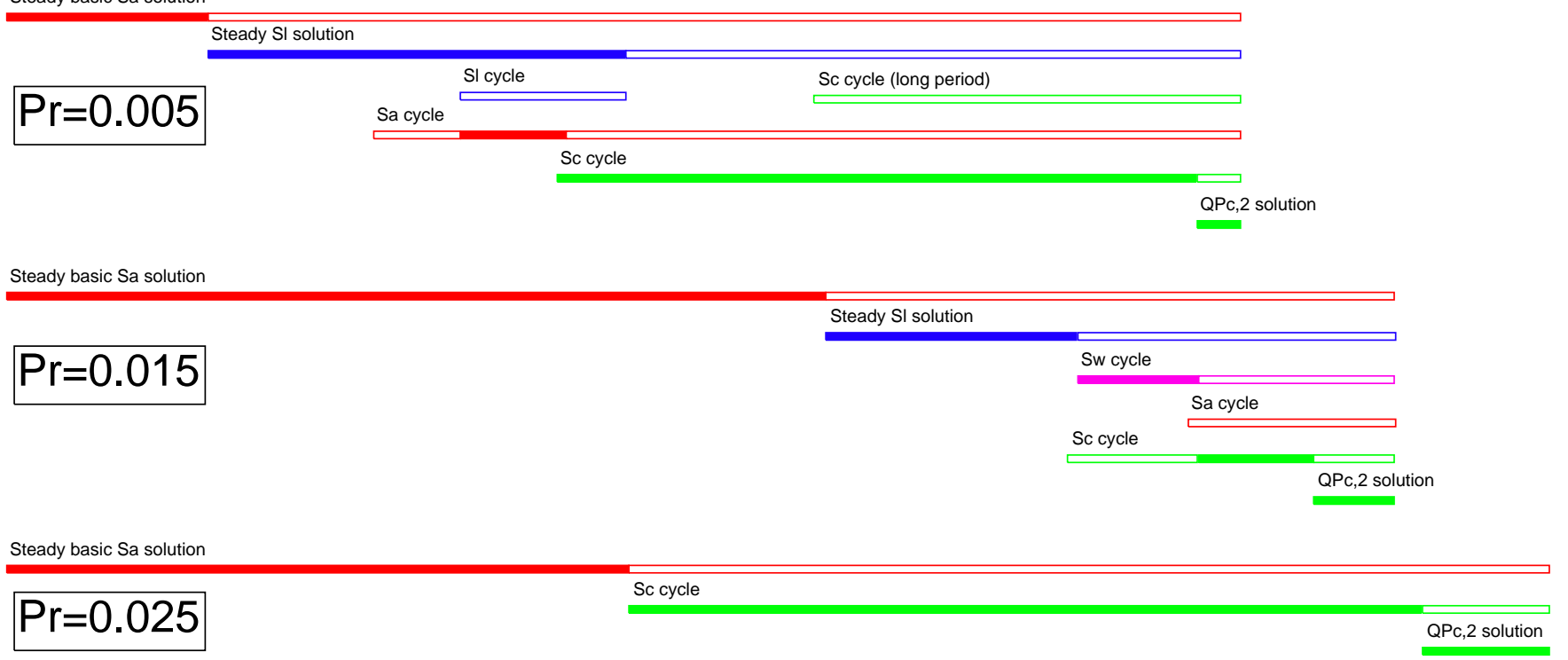




\begin{tabular}{|c|c|c|c|c|c|c|c|c|c|c|}
\hline | Intersection | & $H_{c, 0}^{P} / P_{l}^{P}$ & & $H_{c, 0}^{P} / H_{a}^{P}$ & & $H_{l}^{S} / H_{w, 25}^{S}$ & & $H_{a}^{P} / H_{c, 25}^{P}$ & & $P_{l}^{P} / H_{c, 25}^{P}$ & \\
\hline $\begin{array}{l}P r_{c} \\
G r_{c}\end{array}$ & $\begin{array}{c}8.83 \times 10^{-5} \\
28327\end{array}$ & & $\begin{array}{c}3.28 \times 10^{-4} \\
29398\end{array}$ & & $\begin{array}{l}0.0121 \\
39518\end{array}$ & & $\begin{array}{l}0.0144 \\
37642\end{array}$ & & $\begin{array}{l}0.0163 \\
36630\end{array}$ & \\
\hline $\mid \operatorname{Pr}$ ranges $\mid \mathrm{R} 1$ & | & $\mathrm{R} 2$ & | & R3 & | & $\mathrm{R} 4$ & $\mid$ & R5 & $\mid$ & $\mathrm{R} 6$ \\
\hline
\end{tabular}

Table 3: Intersection points of the paths of the main bifurcation points on the primary and secondary branches; $\operatorname{Pr}$ ranges which are thus defined in the studied interval $0 \leqslant$ $\operatorname{Pr} \leqslant 0.025$.

secondary branch initiated at $P_{l}^{P}$ and of the pitchfork bifurcation of cycles $P_{w}^{C_{c, 25}}$ on the $S_{c}$ cycle initiated at $H_{c, 25}^{P}$. In this range of $P r$, as for $P r=0.015$, we can expect to have a stable secondary branch up to the point $H_{w, 25}^{S}$, where a stable $S_{w}$ cycle is initiated. This $S_{w}$ cycle ends at $P_{w}^{C_{c, 25}}$, where it stabilizes the $S_{c}$ cycle, now unstable at its onset. A quasi-periodic state with $S_{c}$ symmetry will eventually appear when the $S_{c}$ cycle will be destabilized. Note that, in this range of $\operatorname{Pr}$, the $S_{a}$ cycle is expected to remain unstable and to play no role in the transition scenario.

The next changes, when decreasing $P r$, occur at $\operatorname{Pr}_{c}=1.44 \times 10^{-2}$ and $1.21 \times 10^{-2}$ and correspond to the crossings of the $H_{c, 25}^{P}$ and $H_{a}^{P}$ bifurcation points on the primary branch and the $H_{l}^{S}$ and $H_{w, 25}^{S}$ bifurcation points on the secondary branch, respectively. Beyond these two crossings, in the R3 range, the dynamics found in the R5 range and involving the $S_{w}$ cycle and the $S_{c}$ cycle is now entirely unstable as these two cycles are affected by a supplementary unstable complex conjugate mode. As shown in Fig. 8a for $\operatorname{Pr}=0.005$, the important dynamics in this R3 range is connected with the $S_{l}$ and $S_{a}$ cycles: the secondary steady branch is stable up to $H_{l}^{S}$, giving birth subcritically to an unstable $S_{l}$ cycle, which will eventually stabilize the $S_{a}$ cycle at the pitchfork point $P_{l}^{C_{a}}$. Another pitchfork point on the $S_{a}$ cycle will give rise to a stable $S_{c}$ cycle and finally to a $\mathrm{QP}_{c, 2}$ state with central symmetry.

In the intermediate $\mathrm{R} 4$ range, the dynamics must be more complex as, compared to the R5 range, only the crossing of $H_{c, 25}^{P}$ and $H_{a}^{P}$ on the primary branch has occurred. To have more information on the dynamics in this $\mathrm{R} 4$ range, we have calculated the bifurcation diagram for $\operatorname{Pr}=0.013$, which is presented in Fig. 12. We see that this diagram involves both the characteristics found in the R5 and R3 ranges. The $S_{w}$ cycle, stable at onset as in the $\mathrm{R} 5$ range, is now destabilized at a Naimark-Sacker bifurcation $H_{w}^{C_{w, 25}}$, before to disappear at the pitchfork bifurcation $P_{w}^{C_{c, 25}}$ on the $S_{c}$ cycle coming from $H_{c, 25}^{P}$. This $S_{c}$ cycle, now two time unstable at onset, is then not stabilized. In contrast, as in the R3 domain, the $S_{a}$ cycle is only one-time unstable at onset at $H_{a}^{P}$ and is stabilized at the pitchfork bifurcation $P_{l}^{C_{a}}$ to the $S_{l}$ cycle. Note that this $S_{l}$ cycle is two-time unstable at its subcritical onset at $H_{l}^{S}$ and a Naimark-Sacker bifurcation $H_{w}^{C_{l}}$ makes this cycle one-time unstable for smaller $G r$ in order to fit with its stability at $P_{l}^{C_{a}}$. As already seen, the $S_{a}$ cycle is then destabilized at a pitchfork point $\left(G r_{c}=38844\right)$, which gives birth to a secondary $S_{c}$ cycle, slightly sub-critical at its origin, stable on a large domain of $G r$ from $G r_{c}=38767$ to $G r_{c}=43665$. The subsequent scenario is different from what 


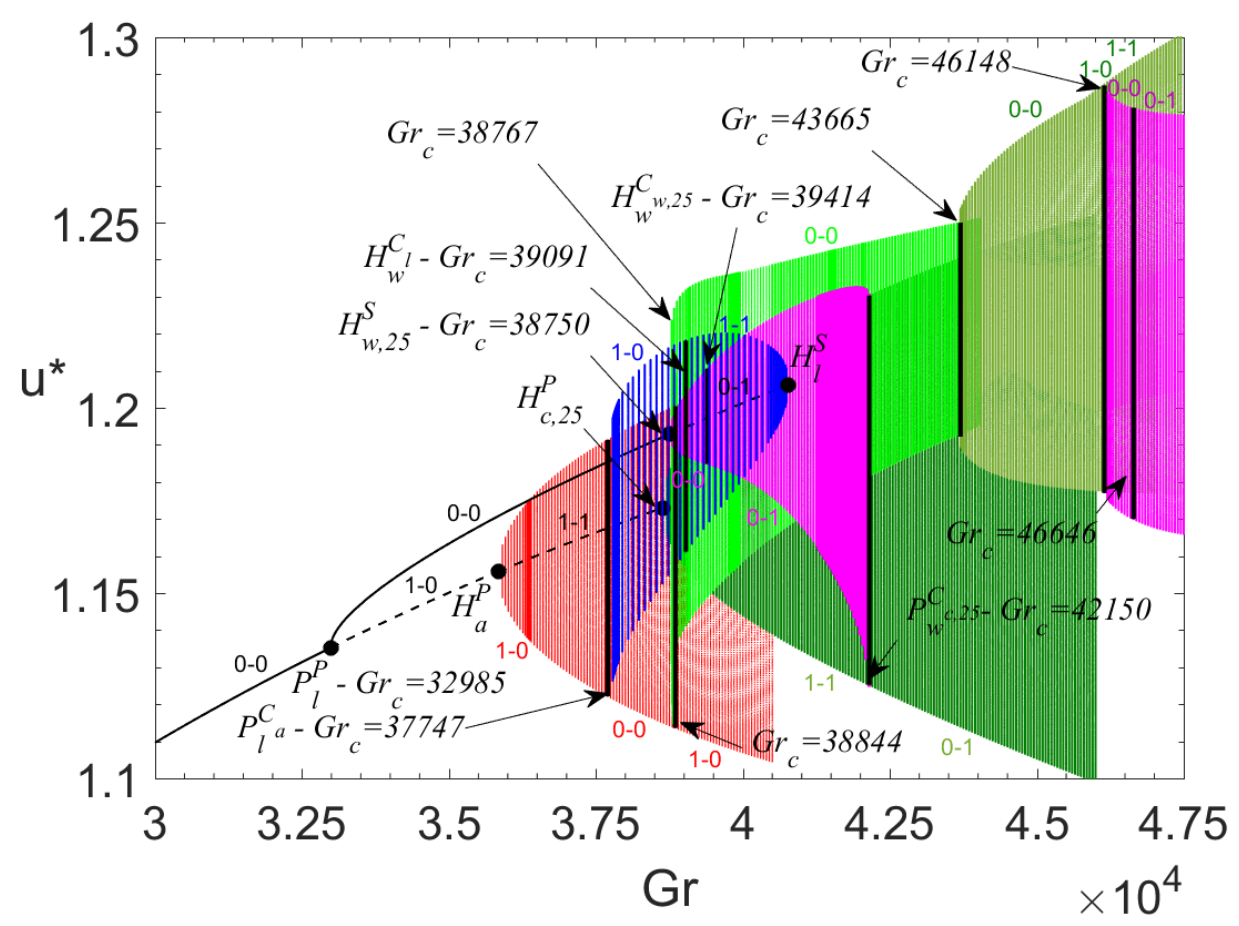

Figure 12: Bifurcation diagram $\left(u^{*}\right.$ versus $\left.G r\right)$ for $\operatorname{Pr}=0.013$ showing all the convective solutions from the stable steady solution with all the symmetries to the appearance of quasi-periodicity. As in the previous bifurcation diagrams, the $S_{a}$ cycle is red, the $S_{l}$ cycle is blue, the $S_{c}$ cycles are green and the $S_{w}$ cycles are pink. The stability of the different states is indicated by the number of unstable eigenvalues $n-m$ (see text in section 4 ). This case is intermediate between the cases at $\operatorname{Pr}=0.015$ and $\operatorname{Pr}=0.005$ and parts of their dynamics are found here. Note the occurrence of a period-doubling bifurcation at $G r_{c}=43665$, a specificity of this case.

was obtained for other values of $\operatorname{Pr}$. The $S_{c}$ cycle is destabilized by a period-doubling bifurcation initiating an $S_{c}$ cycle with a double period. This new $S_{c}$ cycle is destabilized at $G r_{c}=46148$ at a pitchfork bifurcation giving rise to a $S_{w}$ cycle without symmetry stable up to $G r_{c}=46646$ and then to a quasi-periodic state without symmetry, $\mathrm{QP}_{w}$. In fact, on the new $S_{c}$ cycle, there is also a Naimark-Sacker bifurcation very close to 46148 . The $\mathrm{QP}_{c, 3}$ quasi-periodic state with central symmetry which then appears is unstable at onset, but must be further stabilized as it can be obtained by time-stepping calculations at larger $G r$ values as $G r=46300$ and 46600 . There is then the coexistence of both quasi-periodic states without symmetry and with the $S_{c}$ symmetry in a certain range of $G r$ values, as it was confirmed by time-stepping calculations at $G r=46650$.

A specificity of the R4 range is the existence of the Naimark-Sacker bifurcations $H_{w}^{C_{l}}$ and $H_{w}^{C_{w, 25}}$ on the $S_{l}$ and $S_{w}$ cycles, respectively. Some extra calculations have shown that, for $\operatorname{Pr}=0.0142$, these bifurcation points $H_{w}^{C_{l}}$ and $H_{w}^{C_{w, 25}}$ are close to the existence limit $P_{l}^{C_{a}}$ and $P_{w}^{C_{c, 25}}$ of their respective cycles, whereas, for $\operatorname{Pr}=0.0122, H_{w}^{C_{l}}$ and $H_{w}^{C_{w, 25}}$ are close to the birth points $H_{l}^{S}$ and $H_{w, 25}^{S}$ of their respective cycles and also close to each other. All this means that the $H_{w}^{C_{l}}$ and $H_{w}^{C_{w}, 25}$ points appear at $P_{l}^{C_{a}}$ and 
$P_{w}^{C_{c, 25}}$, respectively, when the $H_{a}^{P}$ and $H_{c, 25}^{P}$ bifurcation points cross on the primary branch at $\operatorname{Pr}_{c}=1.44 \times 10^{-2}$ and that they disappear at $H_{l}^{S}$ and $H_{w, 25}^{S}$, when these $H_{l}^{S}$ and $H_{w, 25}^{S}$ points cross on the secondary branch at $\operatorname{Pr}_{c}=1.21 \times 10^{-2}$. This is also an indication that the stable part of the $S_{w}$ cycle, between $H_{w, 25}^{S}$ and $H_{w}^{C_{w, 25}}$, very progressively shortens between $\operatorname{Pr}=1.44 \times 10^{-2}$ and $\operatorname{Pr}=1.21 \times 10^{-2}$ and eventually disappears at $\operatorname{Pr}=1.21 \times 10^{-2}$.

The last crossings of bifurcation points occur on the primary branch, crossing of $H_{c, 0}^{P}$ and $H_{a}^{P}$ at $P r_{c}=3.28 \times 10^{-4}$ and crossing of $H_{c, 0}^{P}$ and $P_{l}^{P}$ at $\operatorname{Pr}_{c}=8.83 \times 10^{-5}$, and they delimit the two ranges R2 and R1 of $\operatorname{Pr}$. Except for the limit case $\operatorname{Pr}=0$, we do not have bifurcation diagrams in these ranges and some extra-calculations will then be necessary to understand the transition between the diagrams at $\operatorname{Pr}=0.005$ and $\operatorname{Pr}=0$. We will follow interesting new bifurcation points appearing on the cycles in these $P r$ ranges (Fig. 13) and compute a newly observed cycle for $\operatorname{Pr}=0.0001$ (Fig. 14). Compared to the diagram at $\operatorname{Pr}=0.005$, the crossing of $H_{c, 0}^{P}$ and $H_{a}^{P}$ at $P r_{c}=3.28 \times 10^{-4}$ will change the stability of the $S_{a}$ cycle at its onset at $H_{a}^{P}$, with one pair of unstable complex conjugate Floquet multipliers in addition to the already unstable real Floquet multiplier. At the same time, however, a new Naimark-Sacker bifurcation $H_{c}^{C_{a}}$ appears on the $S_{a}$ cycle and stabilizes the pair of complex conjugate Floquet multipliers. As shown in Fig. 13, $H_{c}^{C_{a}}$ evolves from $H_{a}^{P}$ at $\operatorname{Pr}=3.28 \times 10^{-4}$, crosses the path of $P_{l}^{C_{a}}$, the other bifurcation on the $S_{a}$ cycle, at $\operatorname{Pr} \approx 2.3 \times 10^{-4}$, before continuing towards $\operatorname{Pr}=0$. The appearance of this $H_{c}^{C_{a}}$ point allows to keep the dynamics obtained at $\mathrm{Pr}=0.005$, i.e. the stabilization of the $S_{a}$ cycle at $P_{l}^{C_{a}}$ and the further dynamics for larger $G r$, at least down to $\operatorname{Pr}=2.3 \times 10^{-4}$. Indeed, for $\operatorname{Pr}$ values below $2.3 \times 10^{-4}$, it is now above $H_{c}^{C_{a}}$ that the $S_{a}$ cycle is stabilized, in coherence with what is observed for $\mathrm{Pr}=0$. Finally, the crossing of $H_{c}^{C_{a}}$ and $P_{l}^{C_{a}}$ also modifies the unstable spectrum of the $S_{l}$ cycle at $P_{l}^{C_{a}}$, where it emerges from the $S_{a}$ cycle, as a pair of complex conjugate Floquet multiplyers is now destabilized. We found, however, that the crossing also induces the creation of a new Naimark-Sacker bifurcation $H_{w}^{C_{l}}$ on the $S_{l}$ cycle, stabilizing these Floquet multiplyers. Beyond $H_{w}^{C_{l}}$, the unstable spectrum of the $S_{l}$ cycle is then the same as before the crossing, allowing to maintain the dynamics on the secondary branch to which the $S_{l}$ cycle is connected at $H_{l}^{S}$. $H_{w}^{C_{l}}$ will move all along the $S_{l}$ cycle, from $P_{l}^{C_{a}}$ at $\operatorname{Pr}=2.3 \times 10^{-4}$ to $H_{l}^{S}$ at $\operatorname{Pr}=8.83 \times 10^{-5}$, i.e. at the crossing of $H_{c, 0}^{P}$ and $P_{l}^{P}$. This last crossing does not change the dynamics initiated from the primary branch and previously described.

We have now to describe the specific dynamics due to the bifurcation point $H_{w, 0}^{S}$ that appears on the secondary branch at the crossing of $H_{c, 0}^{P}$ and $P_{l}^{P}$ at $\operatorname{Pr}_{c}=8.83 \times 10^{-5}$. $H_{w, 0}^{S}$ will destabilize the secondary steady solution in a certain range of $G r$ just above $\operatorname{Pr}=8.83 \times 10^{-5}$ (Fig. 13). This range of $G r$ will shorten as $\operatorname{Pr}$ is increased and disappear at $\operatorname{Pr} \approx 1.1 \times 10^{-4}$. $H_{w, 0}^{S}$ will also generate a stable $S_{w}$ cycle without symmetry and with a long period. This cycle is shown in Fig. 14 for $\operatorname{Pr}=1 \times 10^{-4}$. It is stable from $G r_{c}=28581$, where it is initiated at $H_{w, 0}^{S}\left(f_{c}=2.76\right)$, to $G r_{c}=29705$, where is it destabilized at a Naimark-Sacker bifurcation and gives rise to a quasi-periodic state. This quasi-periodic state without symmetry was never observed as a stable state, meaning that the Naimark-Sacker bifurcation must be subcritical. In contrast, the $\mathrm{QP}_{c, 1}$ state with $S_{c}$ symmetry, which exists and is stable between $H_{c}^{C_{c, 0}}$ and $H_{c}^{C_{a}}$ for $\operatorname{Pr}=0$ and up to $\operatorname{Pr}_{c}=8.83 \times 10^{-5}$ in the $\mathrm{R} 1$ range, continues to be stable in a certain $G r$ range above $\operatorname{Pr}=8.83 \times 10^{-5}$ despite the destabilization of the $S_{c}$ cycle. This $\mathrm{QP}_{c, 1}$ state was observed by time-stepping calculations below $H_{c}^{C_{a}}$ in a $G r$ range decreasing in size with the increase of $\operatorname{Pr}$ and disappearing at the crossing of $H_{c}^{C_{a}}$ and $P_{l}^{C_{a}}$ at $\operatorname{Pr}=2.3 \times 10^{-4}$. 


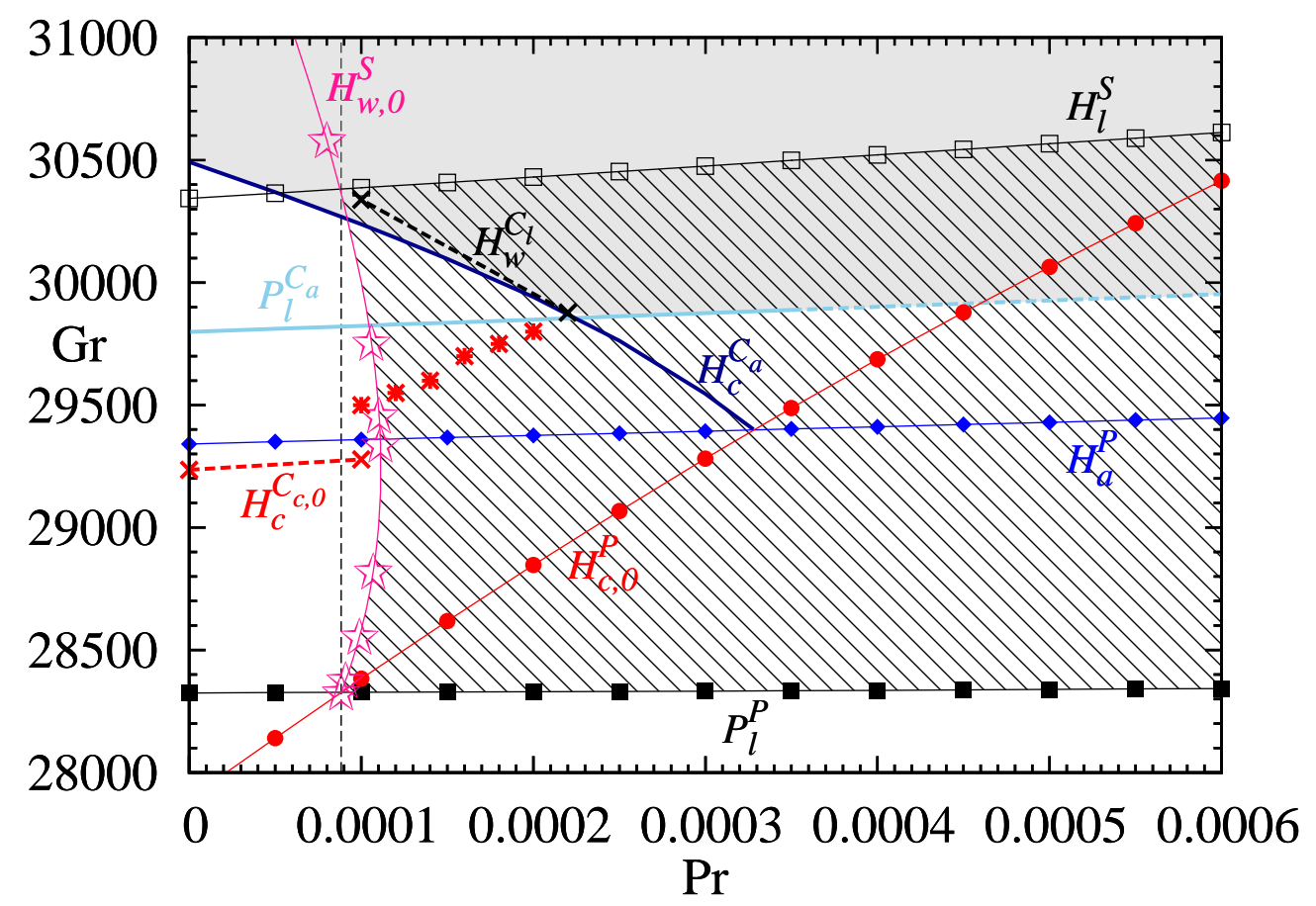

Figure 13: Paths of the bifurcation points in the very small $P r$ range. In addition to the bifurcation points on the primary and secondary steady branches $\left(P_{l}^{P}, H_{c, 0}^{P}, H_{a}^{P}\right.$, $H_{l}^{S}$, and $H_{w, 0}^{S}$, as in Fig. 4b), are given the bifurcation points on the $S_{a}$ cycle, steady bifurcation $P_{l}^{C_{a}}$ (sky-blue line, extrapolated with a dashed part) and Naimark-Sacker bifurcation $H_{c}^{C_{a}}$ (dark-blue line), and the bifurcation $H_{w}^{C_{l}}$ on the $S_{l}$ cycle calculated for $\operatorname{Pr}=1 \times 10^{-4}$ and $\operatorname{Pr}=2.2 \times 10^{-4}$ (black crosses) and interpolated in between (dashed black line). The domains where are stable the secondary steady flow (domain with oblique lines) and the $S_{a}$ cycle (domain with grey backgound) are highlighted. The bifurcation $H_{c}^{C_{c, 0}}$ on the $S_{c, 0}$ cycle is given for $\operatorname{Pr}=0$ and $\operatorname{Pr}=1 \times 10^{-4}$ (red crosses) and interpolated in between (dashed red line). For $P r \leqslant 8.83 \times 10^{-5}$, the $\mathrm{QP}_{c, 1}$ state can be obtained between $H_{c}^{C_{c, 0}}$ and $H_{c}^{C_{a}}$. Above $\operatorname{Pr}=8.83 \times 10^{-5}$ (dashed vertical black line), the $\mathrm{QP}_{c, 1}$ state is still observed in the $G r$ range below $H_{c}^{C_{a}}$ and above the red asterisks, which define an approximate lower limit.

This $G r$ range can be seen in Fig. 14 between the $H_{c}^{C_{a}}$ curve and the red asterisks, which give the smallest $G r$ values where the $\mathrm{QP}_{c, 1}$ state was obtained. Note finally that the second point $H_{w, 0}^{S}$ appearing at $G r_{c}=30004$ on the secondary branch, and restabilizing it, does not give rise to stable states as it can be observed in Fig. 14 for $\operatorname{Pr}=1 \times 10^{-4}$. From all these results obtained for the very low values of $P r$, we can also conclude that the dynamics in the whole $\mathrm{R} 1$ range is similar to that obtained for $\operatorname{Pr}=0$, with only some variations of the main bifurcation points, $H_{c, 0}^{P}$, the onset of the stable $S_{c}$ cycle with long period, $H_{c}^{C_{c, 0}}$, the onset of the stable $\mathrm{QP}_{c, 1}$ state and $H_{c}^{C_{a}}$, the limit of the $\mathrm{QP}_{c, 1}$ state and onset of the stable $S_{a}$ cycle (see Fig. 13).

We finally give a summary of all the different stable flow states, from steady to quasiperiodic, which have been obtained in the study for $0 \leqslant \operatorname{Pr} \leqslant 0.025$ in Table 4 . We also 


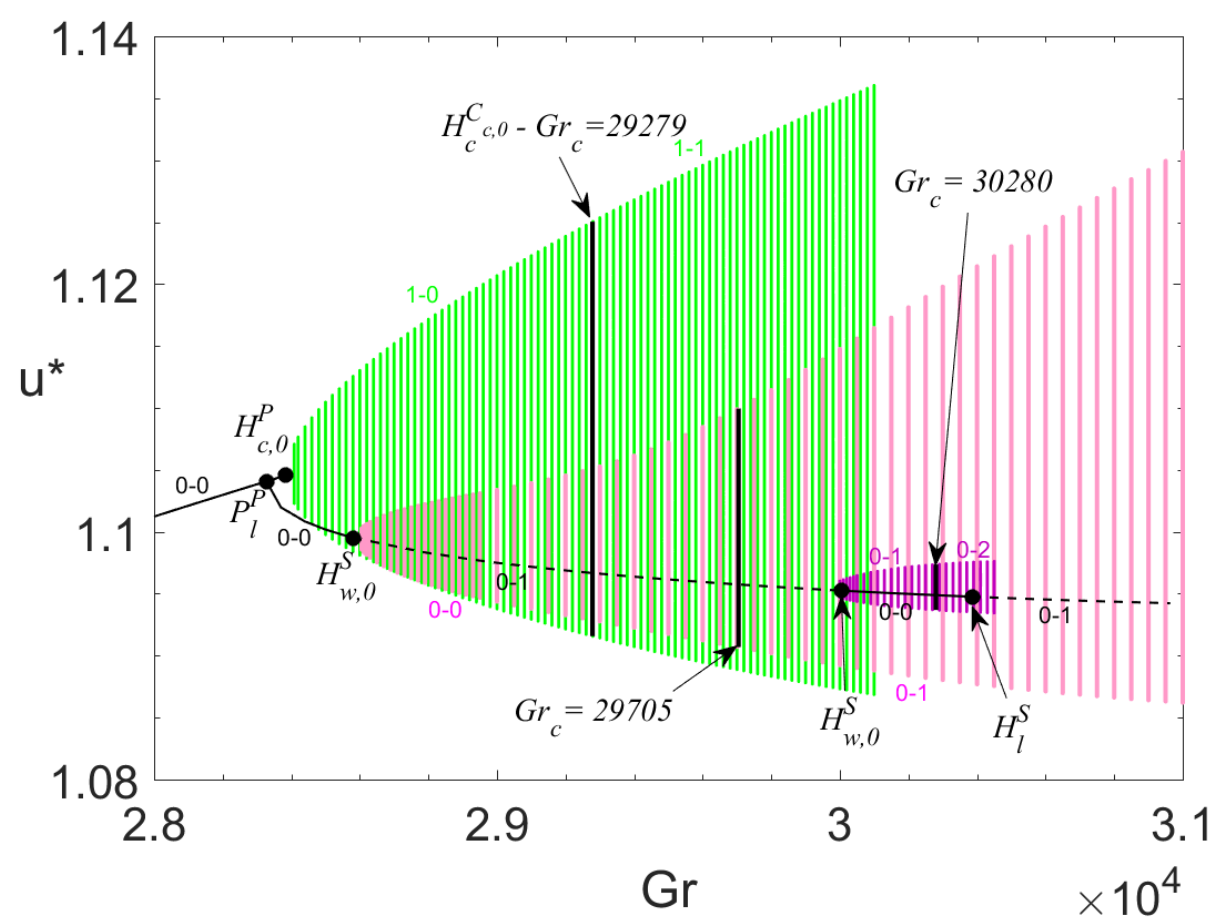

Figure 14: Bifurcation diagram ( $u^{*}$ versus $G r$ ) for $\operatorname{Pr}=1 \times 10^{-4}$ highlighting the $S_{c}$ cycle with long period (green color) initiated at $H_{c, 0}^{P}\left(G r_{c}=28383, f_{c}=2.79\right)$ on the primary branch and unstable at this value of $\operatorname{Pr}$ and the $S_{w}$ cycles with long periods (pink colors) initiated at the two points on the secondary branch belonging to the $H_{w, 0}^{S}$ path. The $S_{w}$ cycle initiated at $G r_{c}=28581$ (light-pink color, $f_{c}=2.76$ ) is stable at onset and then destabilized at $G r_{c}=29705$ at a Naimark-Sacker bifurcation, whereas the $S_{w}$ cycle initiated at $G r_{c}=30004\left(f_{c}=2.20\right)$ remains unstable. The steady secondary branch is stable from $P_{l}^{P}\left(G r_{c}=28328\right)$ to the first $H_{w, 0}^{S}$ point, and then from the second $H_{w, 0}^{S}$ point to $H_{l}^{S}\left(G r_{c}=30386\right)$.

indicate in which domain of $\operatorname{Pr}$ from R1 to R6 (see table 3) they can be found. Fourteen different stable states have been obtained, corresponding to two steady states, eight cycles and four quasi-periodic states. We see that, besides the basic $S_{a}$ steady state, the quasiperiodic $\mathrm{QP}_{c, 2}$ state is found in all the $\operatorname{Pr}$ ranges. The primary $S_{c}$ cycle is stable for the largest values of $\mathrm{Pr}$ in ranges $\mathrm{R} 5$ and $\mathrm{R} 6$, whereas the primary $S_{a}$ cycle, although never stable at its onset, is obtained as a stable state in the other ranges R4 to R1 and gives birth to a stable secondary $S_{c}$ cycle. The secondary $S_{w}$ cycle is stable in R 5 where it also stabilizes the primary $S_{c}$ cycle, but also in R4 in a shrinking interval of $G r$. In contrast, the secondary $S_{l}$ cycle is never obtained as a stable state, although it is involved in the stabilisation of the $S_{a}$ cycle. The long period primary $S_{c}$ cycle is only stable in R1, but another long period secondary $S_{w}$ cycle is stable in a very small range of $\operatorname{Pr}$ inside R2. The quasi periodic $\mathrm{QP}_{c, 1}$ state, stable in $\mathrm{R} 1$ and part of $\mathrm{R} 2$, has the peculiarity of being an intermediate state between stable cycles. Finally, the largest number of stable states is obtained in R4, which appears as a range of $\operatorname{Pr}$ where transition between different bifurcation scenarios occurs. 


\begin{tabular}{|c|c|c|c|c|c|c|}
\hline $\operatorname{Pr}$ ranges & | R1 & $\mathrm{R} 2$ & R3 & $\mathrm{R} 4$ & R5 & R6 \\
\hline Basic $S_{a}$ steady state & $\times$ & $\times$ & $\times$ & $\times$ & $\times$ & $\times$ \\
\hline Secondary $S_{l}$ steady state & & $\times$ & $\times$ & $\times$ & $\times$ & \\
\hline Primary $S_{c}$ cycle & & & & & $\times$ & $\times$ \\
\hline Primary $S_{c}$ cycle (long period) & $\times$ & & & & & \\
\hline Primary $S_{a}$ cycle & $\times$ & $\times$ & $\times$ & $\times$ & & \\
\hline Secondary $S_{w}$ cycle & & & & $\times$ & $x$ & \\
\hline Secondary $S_{c}$ cycle & $\times$ & $\times$ & $\times$ & $\times$ & & \\
\hline Secondary $S_{w}$ cycle (long period) & & $\times$ & & & & \\
\hline Secondary $S_{c}$ cycle (double period) & & & & $\times$ & & \\
\hline Secondary $S_{w}$ cycle (double period) & & & & $\times$ & & \\
\hline $\mathrm{QP}_{c, 1}$ state & $x$ & $\times$ & & & & \\
\hline $\mathrm{QP}_{c, 2}$ state & $\times$ & $\times$ & $x$ & $\times$ & $x$ & $\times$ \\
\hline $\mathrm{QP}_{c, 3}$ state & & & & $\times$ & & \\
\hline $\mathrm{QP}_{w}$ state & & & & $\times$ & & \\
\hline
\end{tabular}

Table 4: Summary of the different stable flow states, from steady to quasi-periodic, which have been obtained in the study and the domains of $\mathrm{Pr}$ from R1 to R6 (see table 3) where they can be found. The cycles are given as primary when they are initiated at a Hopf bifurcation on the steady primary branch and as secondary when they are initiated at a Hopf bifurcation on the steady secondary branch or at a pitchfork bifurcation on another cycle.

\section{Conclusion}

Buoyant convection is an ubiquitous phenomenon both in nature and industry. This is why it has been considered as a classical problem of physical fluid mechanics for a long time. Our interest in this problem is motivated by crystal growth applications where oscillatory convection often rises in the liquid bulk subjected to a horizontal temperature gradient and highly impacts the quality of the final solid product. Moreover, the numerical studies previously concerned by this problem mainly used time-stepping approaches for selected cases and could not catch the precise dynamics involved in the transition from steady to periodic and quasi-periodic states.

The numerical study presented in this paper uses powerful continuation techniques allowing to follow both steady and periodic solutions when the governing parameter is varied. It is focused on a side heated parallelepipedic cavity of given aspect ratios $\left(A_{X}=4, A_{Y}=2\right)$, filled with a low-Prandtl number fluid in the range $0 \leqslant \operatorname{Pr} \leqslant 0.025$. Despite this very small $\operatorname{Pr}$ range, our results have shown a complex flow dynamics, with bifurcation scenarios involving different steady and periodic solutions and changing with $\operatorname{Pr}$. Rather than giving a summary of all the bifurcation scenarios observed, we will quote important information obtained during this study.

In these situations heated from the side and at small $\mathrm{Pr}$, the steady solutions are only few: the basic one-roll flow with all the symmetries (basic steady branch) and, possibly, another solution corresponding to the breaking of one of the symmetries (here the $S_{l}$ solution on a secondary steady branch). In contrast, the oscillatory periodic solutions are numerous, initiated at the different Hopf bifurcation points on the primary or secondary steady branches, and several of these cycles are involved in the dynamics of the system leading to the stable solutions. If the scenario is simple for the largest values of $\operatorname{Pr}$ (as 
$\operatorname{Pr}=0.025)$ with a supercritical Hopf bifurcation on the primary branch leading to a periodic solution, which is stable in a large range of $G r$, the situation becomes more complex for smaller values of $P r$. In particular, in the domain of $P r$ where the first instability is steady and where a stable secondary steady branch exists, some cycles that are unstable at their onset on the primary branch will be stabilized by interaction with cycles coming from the secondary branch. A striking example is for example given by the case $\operatorname{Pr}=0.005$ : the secondary branch is stable up to a Hopf bifurcation with $S_{l}$ symmetry and a stable $S_{l}$ cycle could then be expected. In fact, the Hopf bifurcation being subcritical, the $S_{l}$ cycle is unstable and cannot be observed, but it stabilizes a $S_{a}$ cycle which can then be obtained, together with a $S_{c}$ cycle which bifurcates from the $S_{a}$ cycle. We can also note the possible coexistence of stable solutions (steady or oscillatory) in certain $G r$ ranges. The situations closer to $\operatorname{Pr}=0$ are particularly complex because the bifurcation points on the steady primary and secondary branches co-exist in a smaller $G r$ range. And the scenario obtained for $P r=0$, which, in the past, has been thought to be representative of what is obtained in the small $\mathrm{Pr}$ range, is in fact strictly valid only for $\operatorname{Pr} \leqslant 8.83 \times 10^{-5}$.

This study has also shown that the bifurcation scenarios change at the different intersections of the bifurcation points on the primary and secondary steady branches, but also sometimes at intersections of bifurcation points on cycles. We have tried to understand all the changes induced on the dynamics by these different intersections and then to give a clear view of the dynamics in the whole $\mathrm{Pr}$ range considered. In any case, the bifurcation scenarios, which begin with the basic steady flow, eventually lead to a cycle with $S_{c}$ symmetry and a further quasi-periodic state with the same $S_{c}$ symmetry. An exception was found for $\operatorname{Pr}=0.013$ with a period-doubling bifurcation first affecting the $S_{c}$ cycle and the further coexistence of quasi-periodic states with $S_{c}$ symmetry and without symmetry. This specific dynamics at high $G r$ values could exist in the vicinity of $\operatorname{Pr}=0.013$, but was not observed for $\operatorname{Pr}=0.005$ and $\operatorname{Pr}=0.015$.

Qualitative comparisons can be made with the experiment of Braunsfurth \& Mullin (1996) in a $4 \times 1.3 \times 1$ cavity. In this experiment using liquid gallium, both the Grashof number and the Prandtl number were changed by varying the temperature difference as well as the applied mean temperature. We can note that the cavity is more transversally confined than our cavity, but also that, as shown by Juel et al. (1999), the generated flow has not the $S_{\pi}$ symmetry due to the imperfect insulation of the cavity. A consequence of this last point is that the steady bifurcation leading to the breaking of this $S_{\pi}$ symmetry cannot be found in the experiment. Interestingly, however, different oscillatory states are found in the experiment when varying $G r$ and $P r$, which compares well with our results. The mentionned dimensionless frequencies are 52.1, 22.1, 65.7 and 39.2, values a little higher than our values, but in the same range for $\mathrm{Pr}$ corresponding to gallium. Note also that the experimental temperature measurements were performed through a lid in the $V_{l}$ symmetry plane: a consequence is that, for oscillations that break the leftright symmetry, the temperature signal obtained has twice the frequency of the signals measured elsewhere in the cavity (Hof et al. 2004). The frequencies of the experimental oscillatory flow could then be, in some cases, half the values given. More quantitative comparisons are difficult due to the differences in the geometry and symmetry properties, but also because of the different ways to span the $(G r, P r)$ parameter space.

Our study was limited to $G r$ values allowing to reach quasi-periodic states, a domain where our continuation techniques were particularly useful to understand the flow dynamics. We have not explored the domain of larger $G r$, which would lead to more complex oscillatory flows and eventually to chaotic states, but only time-stepping techniques can be used for such further studies. Our continuation techniques, in contrast, could be 
used to study the flow dynamics in cavities with different aspect ratios. According to Henry \& Ben Hadid (2007), the first instability affecting the basic steady flow strongly depends on the cavity aspect ratios, and can be steady or oscillatory and with different symmetries. In particular, bifurcations leading to states with the $S_{\pi}$ symmetry (never seen in this study) can be obtained for certain aspect ratios. Such changes in the first instability characteristics would imply changes in the bifurcation scenarios, which it could be interesting to study in the future.

\section{Acknowledgements}

This collaborative work was supported by the PHC Maghreb Partnership Program No. 36951NG. A grant of the PROFAS B+ program given to A. M. is also gratefully acknowledged. 


\section{REFERENCES}

Afrid, M. \& ZeBib, A. 1990 Oscillatory three-dimensional convection in rectangular cavities and enclosures. Phys. Fluids 2 (8), 1318-1327.

Bacharoudis, E., Vrachopoulos, M. Gr., Koukou, M. K., Margaris, D., Filios, A. E. \& Mavrommatis, S. A. 2007 Study of the natural convection phenomena inside a wall solar chimney with one wall adiabatic and one wall under a heat flux. Applied Thermal Engineering 27 (13), 2266 - 2275.

Ben Hadid, H. \& Henry, D. 1997 Numerical study of convection in the horizontal Bridgman configuration under the action of a constant magnetic field. Part 2: Three dimensional flow. J. Fluid Mech. 333, 57-83.

Bergeon, A., Henry, Daniel, Ben Hadid, H. \& Tuckerman, L. S. 1998 Marangoni convection in binary mixtures with Soret effect. J. Fluid Mech. 375, 143-177.

Braunsfurth, Manfred G. \& Mullin, T. 1996 An experimental study of oscillatory convection in liquid gallium. J. Fluid Mech. 327, 199-219.

Cormack, D. E., Leal, L.G. \& Imberger, J. 1974a Natural convection in a shallow cavity with differentially heated end walls. Part 1. Asymptotic theory. J. Fluid Mech. 65 (2), 209-229.

Cormack, D. E., Leal, L.G. \& Seinfeld, J. H. 1974b Natural convection in a shallow cavity with differentially heated end walls. Part 2. Numerical solutions. J. Fluid Mech. 65 (2), 231-246.

Daviaud, F. \& Vince, J. M. 1993 Traveling waves in a fluid layer subjected to a horizontal temperature gradient. Phys. Rev. E 48, 4432-4436.

Dhanaraj, G., Byrappa, K., Prasad, V. \& Dudley, M. 2010 Springer Handbook of Crystal Growth. Springer Berlin Heidelberg.

Dupont, S., Marchal, J. M., Crochet, M. \& Geyling, F. T. 1987 Numerical simulation of the horizontal Bridgman growth. Part II: Three-dimensional flow. Int. J. Numer. Methods Fluids 7 (1), 49-67.

Gershuni, G., Laure, P., Myznikov, V., Roux, B. \& Zhukhovitsky, E. 1992 On the stability of plane-parallel advective flows in long horizontal layers. Microgravity Q. 2, $141-151$.

HARt, J. E. 1972 Stability of thin non-rotating Hadley circulations. J. Atmos. Sci. 29, 687-696.

HenRY, D. \& Ben HADID, H. 2007 Multiple flow transitions in a box heated from the side in low-Prandtl-number fluid. Phys. Rev. E 76, 016314.

Henry, D. \& Buffat, M. 1998 Two- and three-dimensional numerical simulations of the transition to oscillatory convection in low-Prandtl-number fluids. J. Fluid Mech. 374, $145-171$.

Hof, B., Juel, A., Zhao, L., Henry, D., Ben Hadid, H. \& Mullin, T. 2004 On the onset of oscillatory convection in molten gallium. J. Fluid Mech. 515, 391-413.

Hung, M. C. \& Andereck, C. D. 1988 Transitions in convection driven by a horizontal temperature gradient. Physics Letters A 132 (5), 253 - 258.

Hurle, D. T. J. 1966 Temperature oscillations in molten metals and their relationship to the growth state in melt grown crystals. Phil. Mag. 13, 305-310.

Hurle, D. T. J., Jakeman, E. \& Johnson, C. P. 1974 Convective temperature oscillations in molten gallium. J. Fluid Mech. 64, 565-576.

Juel, A., Mullin, T., Ben Hadid, H. \& Henry, D. 1999 Magnetohydrodynamic convection in molten gallium. J. Fluid Mech. 378, 97-118.

Juel, A., Mullin, T., Ben Hadid, H. \& Henry, D. 2001 Three-dimensional free convection in molten gallium. J. Fluid Mech. 436, 267-281.

KARNiADAKIS, G. E. 1991 High-order splitting methods for incompressible Navier-Stokes equations. J. Comp. Physics 97, 414-443.

Klausmeier, C. A. 2008 Floquet theory: a useful tool for understanding nonequilibrium dynamics. Theor. Ecol. 1, 53-161.

LAPPA, MARCELlo 2007 Secondary and oscillatory gravitational instabilities in canonical threedimensional models of crystal growth from the melt. Part 2: Lateral heating and the Hadley circulation. Comptes Rendus Mécanique 335 (5), 261 - 268. 
LAure, P. 1987 Étude des mouvements de convection dans une cavité rectangulaire soumise à un gradient de température horizontal. J. Theo. App.Mechanics 6, 351-382.

Laure, P. \& Roux, B. 1989 Linear and non-linear analysis of the Hadley circulation. J. Cryst. Growth 97, 226-234.

Lyubimova, T. P., Lyubimov, D. V., Morozov, V. A., Henry, D. \& Ben Hadid, H. 2009b Stability of convection in a horizontal channel subjected to a longitudinal temperature gradient. Part 2. Effect of a magnetic field. J. Fluid Mech. 635, 297-319.

Lyubimova, T. P., Lyubimov, D. V., Morozov, V. A., Scuridin, R. V., Ben Hadid, H. \& HENRY, D. 2009a Stability of convection in a horizontal channel subjected to a longitudinal temperature gradient. Part 1. Effect of aspect ratio and Prandtl number. J. Fluid Mech. 635, 275-295.

Mamun, Chowdhury K. \& Tuckerman, Laurette S. 1995 Asymmetry and Hopf bifurcation in spherical Couette flow. Phys. Fluids 7 (1), 80-91.

McKell, K. E., Broomhead, D. S., Jones, R. \& Hurle, D. T. J. 1990 Torus doubling in convecting molten gallium. Europhysics Letters 12 (6), 513.

Mercader, I., Batiste, O., Ramírez-Piscina, L., Ruiz, X., Rüdiger, S. \& Casademunt, J. 2005 Bifurcations and chaos in single-roll natural convection with low Prandtl number. Phys. Fluids 17 (10), 104108.

Mohamad, A. A. \& Viskanta, R. 1991 Transient natural convection of low-Prandtl-number fluids in a differentially heated cavity. Int. J. Num. Meth. Fluids 13 (1), 61-81.

Pimputkar, S. M. \& Ostrach, S. 1981 Convective effects in crystals grown from melts. J. Cryst. Growth 55, 614-646.

Puigjaner, D., Herrero, J., Simó, C. \& Giralt, F. 2011 From steady solutions to chaotic flows in a Rayleigh-Bénard problem at moderate Rayleigh numbers. Physica D: Nonlinear Phenomena 240 (11), 920 - 934.

Pulicani, J. P., Arco, E. C. Del, Randriamampianina, A., Bontoux, P. \& Peyret, R. 1990 Spectral simulations of oscillatory convection at low Prandtl number. Int. J. Num. Meth. Fluids 10 (5), 481-517.

Sánchez, J., Net, M., García-Archilla, B. \& Simó, C. 2004 Newton-Krylov continuation of periodic orbits for Navier-Stokes flows. J. Comp. Physics 201 (1), 13 - 33.

Seydel, R. 2010 Practical Bifurcation and Stability Analysis. Springer.

Torres, J. F., Henry, D., Komiya, A. \& Maruyama, S. 2014 Bifurcation analysis of steady natural convection in a tilted cubical cavity with adiabatic sidewalls. J. Fluid Mech. 756, $650-688$.

Torres, J. F., Henry, D., Komiya, A., Maruyama, S. \& Ben Hadid, H. 2013 Threedimensional continuation study of convection in a tilted rectangular enclosure. Phys. Rev. E 88, 043015 .

WAKITANi, S. 2000 Numerical study of three-dimensional oscillatory natural convection at low Prandtl number in rectangular enclosures. J. Heat Transfer 123, 77-83. 